\title{
Removal of Alkyl Sulfonates Using DABCO
}

Kaitlyn Corazzata, Peter J. Rose, Shunyan Mo $\mathrm{M}^{\dagger}$ Joseph Snodgrass, Alexander Langston, Elaine

C. Lee*

\section{Supporting Information}

1. Stability

1.1. Reaction conditions

1.2. Acidic Diluent

1.3. HPLC Internal Standard

2. Kinetic Rate Constants

3. \% Removal Data

4. NMR Characterization of Alkylated DABCO Species

5. LC/MS Data for Removal of Alkylated DABCO with Aqueous Washes

General. Abbreviations

Methyl methanesulfonate (MMS)

Ethyl methane sulfonate (EMS)

n-propyl methanesulfonate (nPMS)

$i$-propyl methanesulfonate (iPMS)

Methyl benzenesulfonate (MBS)

Ethyl benzenesulfonate (EBS)

$n$-propyl benzenesulfonate (nPBS)

$i$-propyl benzenesulfonate (iPBS)

\section{Stability}

\subsection{Reaction conditions}


Various reaction conditions were set up with $0.005 \mathrm{M}$ stock solution of alkyl sulfonates in 2-MeTHF in order to stress test conditions to be used for screening experiments. Condition 4 was chosen as it demonstrated no degradation of sulfonates over time and was simple to set up on vial scale.

Table S1. Stability Experiment Setup Reaction Conditions

\begin{tabular}{|c|c|c|c|}
\hline Experiment & Temp & Atmosphere & Water \\
\hline \hline 1 & 25 & Air & None \\
\hline 2 & 25 & Nitrogen & None \\
\hline 3 & 25 & Nitrogen & $1 \mathrm{wt} \%$ \\
\hline $4^{*}$ & 50 & Air & None \\
\hline 5 & 50 & Nitrogen & None \\
\hline 6 & 50 & Nitrogen & $1 \mathrm{wt} \%$ \\
\hline
\end{tabular}

$*=$ condition used in all subsequent screening experiments.

Table S2. Reaction Conditions Stability Data

\begin{tabular}{|c|c|c|c|c|c|c|c|c|c|}
\hline \multirow{2}{*}{$\begin{array}{c} \\
\text { Time } \\
\text { (h) }\end{array}$} & \multirow[b]{2}{*}{ Experiment } & \multicolumn{8}{|c|}{ Amount of sulfonate remaining (mg) } \\
\hline & & MMS & EMS & nPMS & iPMS & MBS & EBS & nPBS & iPBS \\
\hline \multirow{6}{*}{0} & 1 & 2.20 & 2.63 & 2.68 & 2.80 & 3.34 & 3.56 & 1.80 & 3.65 \\
\hline & 2 & 2.16 & 2.63 & 2.68 & 2.79 & 3.33 & 3.51 & 1.79 & 3.62 \\
\hline & 3 & 2.29 & 2.80 & 2.85 & 2.99 & 3.56 & 3.76 & 1.91 & 3.93 \\
\hline & 4 & 2.32 & 2.81 & 2.88 & 3.01 & 3.56 & 3.75 & 1.91 & 3.90 \\
\hline & 5 & 2.25 & 2.73 & 2.79 & 2.92 & 3.44 & 3.63 & 1.84 & 3.60 \\
\hline & 6 & 1.94 & 2.35 & 2.39 & 2.50 & 2.93 & 3.04 & 1.53 & 3.08 \\
\hline \multirow{6}{*}{5} & 1 & 1.95 & 2.58 & 2.64 & 2.73 & 2.66 & 3.53 & 1.62 & 3.56 \\
\hline & 2 & 1.39 & 1.69 & 1.65 & 1.55 & 1.93 & 2.18 & 0.93 & 1.61 \\
\hline & 3 & 2.30 & 2.85 & 2.91 & 3.03 & 3.61 & 4.42 & 1.81 & 3.97 \\
\hline & 4 & 1.95 & 2.42 & 2.43 & 2.49 & 3.03 & 3.67 & 1.50 & 3.15 \\
\hline & 5 & 2.34 & 2.94 & 2.99 & 3.15 & 3.73 & 4.05 & 1.86 & 4.15 \\
\hline & 6 & 2.19 & 2.72 & 2.77 & 2.90 & 3.47 & 3.77 & 1.73 & 3.74 \\
\hline \multirow{2}{*}{24} & 1 & 1.46 & 1.77 & 1.73 & 1.65 & 2.06 & 2.40 & 1.01 & 1.75 \\
\hline & 2 & 2.21 & 2.76 & 2.82 & 2.93 & 3.51 & 3.79 & 1.78 & 3.78 \\
\hline
\end{tabular}




\begin{tabular}{|c|c|c|c|c|c|c|c|c|c|}
\hline 3 & 2.11 & 2.61 & 2.66 & 2.72 & 3.33 & 3.61 & 1.70 & 3.50 \\
\cline { 2 - 9 } & 4 & 2.28 & 2.85 & 2.92 & 3.09 & 3.66 & 3.96 & 1.87 & 4.06 \\
\cline { 2 - 9 } & 5 & 2.37 & 2.95 & 3.02 & 3.17 & 3.77 & 4.10 & 1.94 & 4.13 \\
\cline { 2 - 9 } & 6 & 2.38 & 2.86 & 2.93 & 3.07 & 3.65 & 3.94 & 1.91 & 4.00 \\
\hline
\end{tabular}

Figure S1. Experiment 1 Stability vs. Time

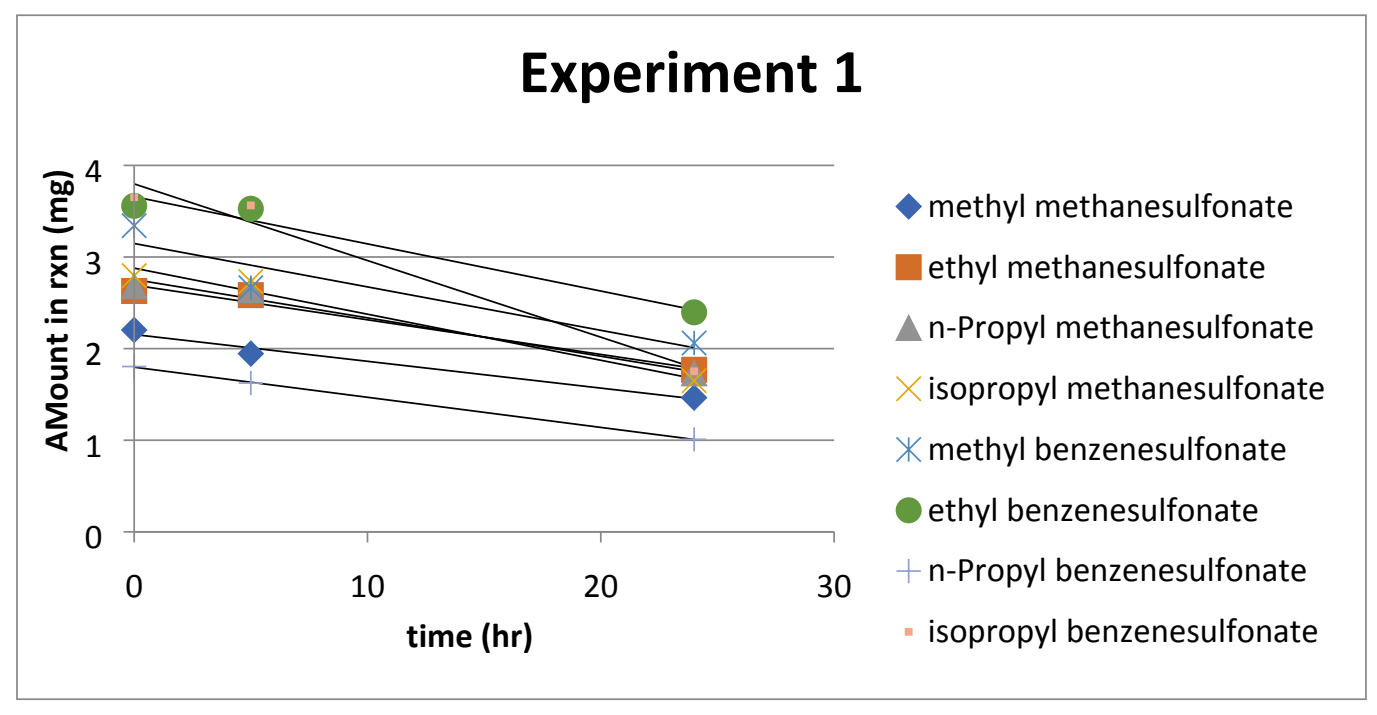

Figure S2. Experiment 2 Stability vs. Time

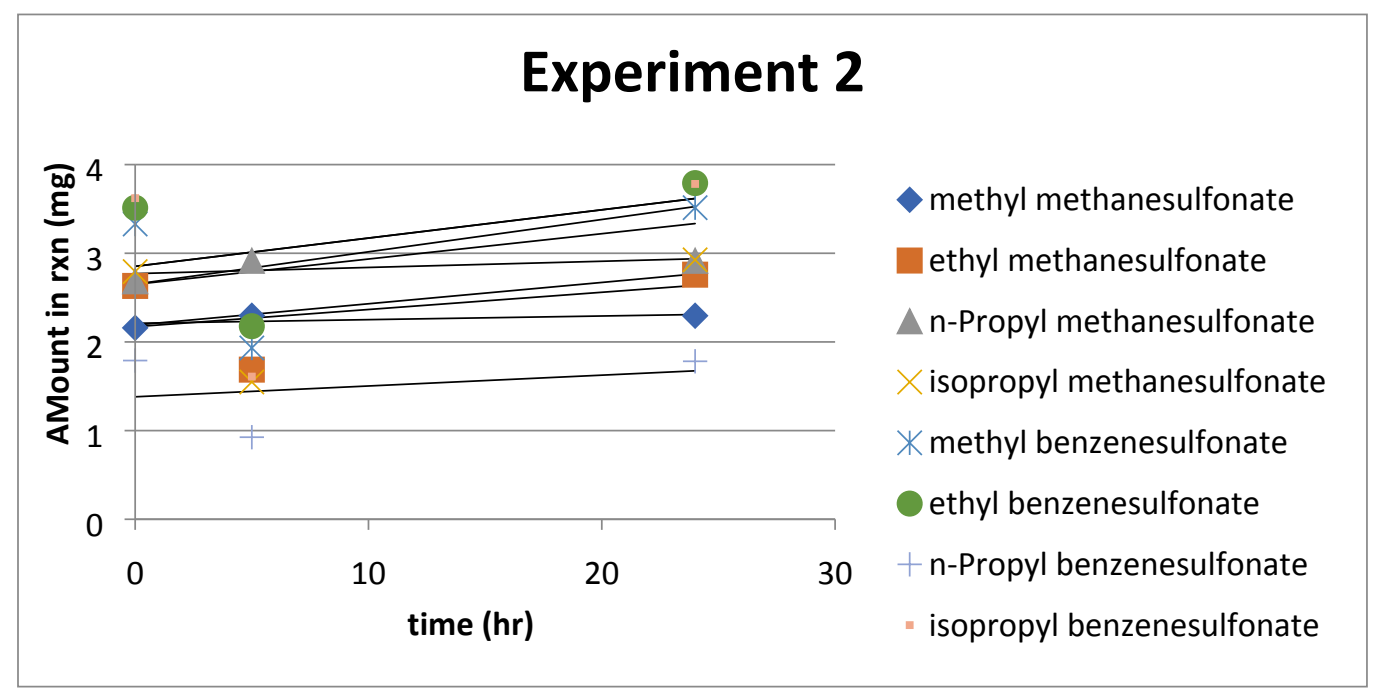


Figure S3. Experiment 3 Stability vs. Time

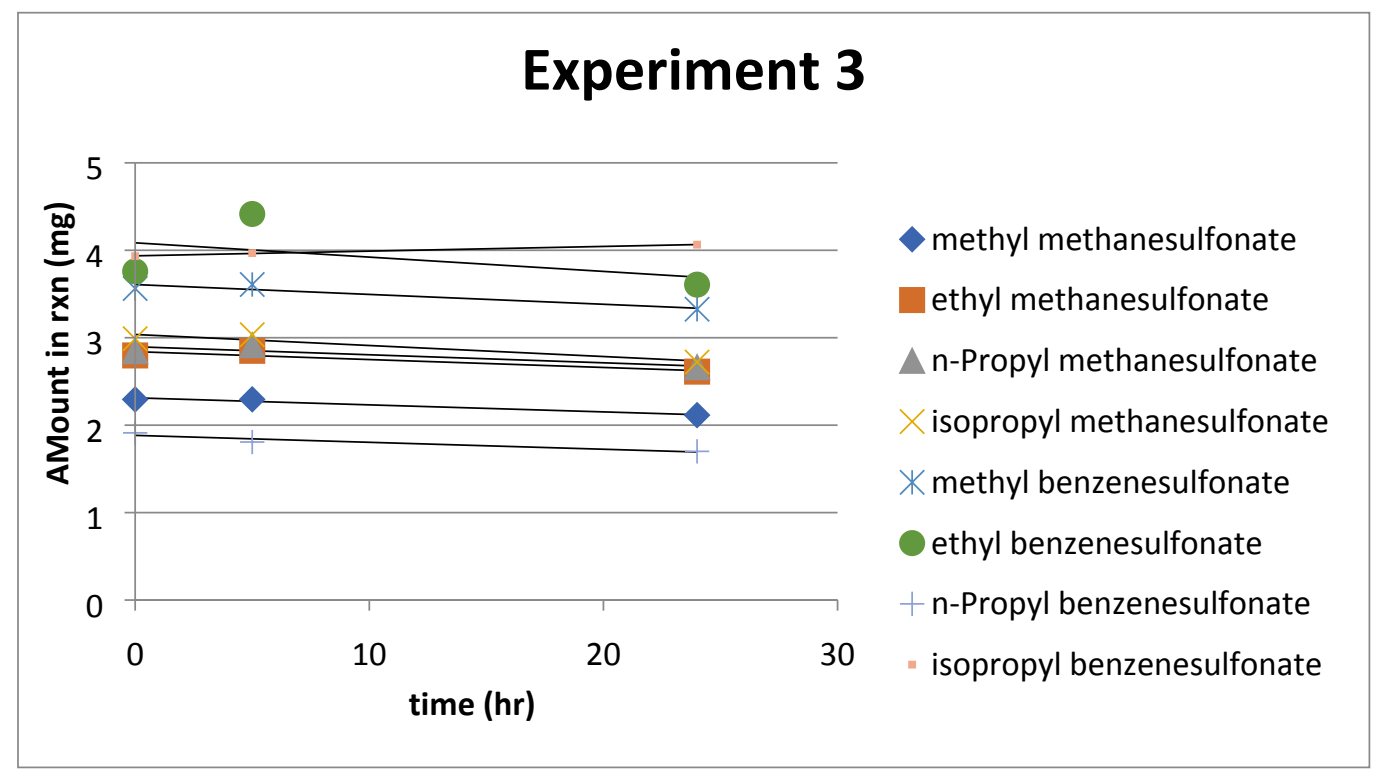

Figure S4. Experiment 4 Stability vs. Time

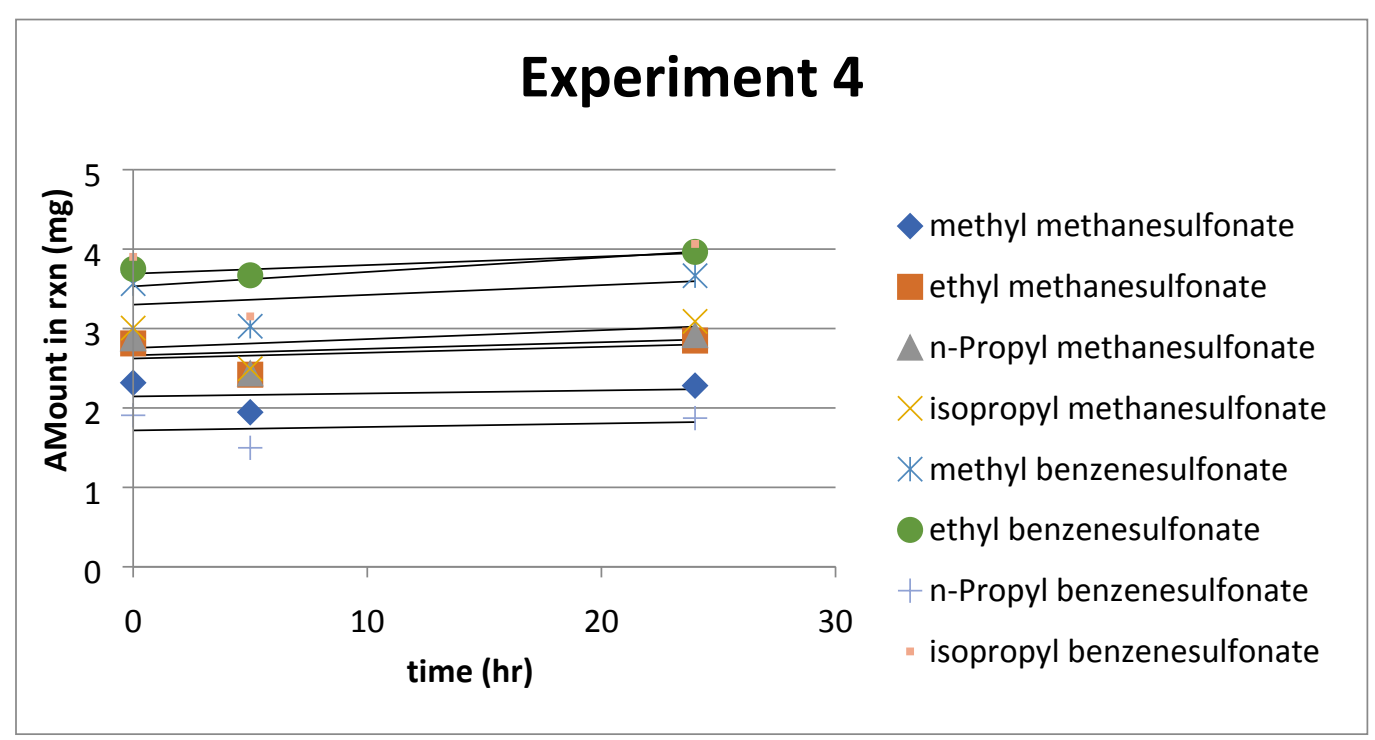


Figure S5. Experiment 5 Stability vs. Time

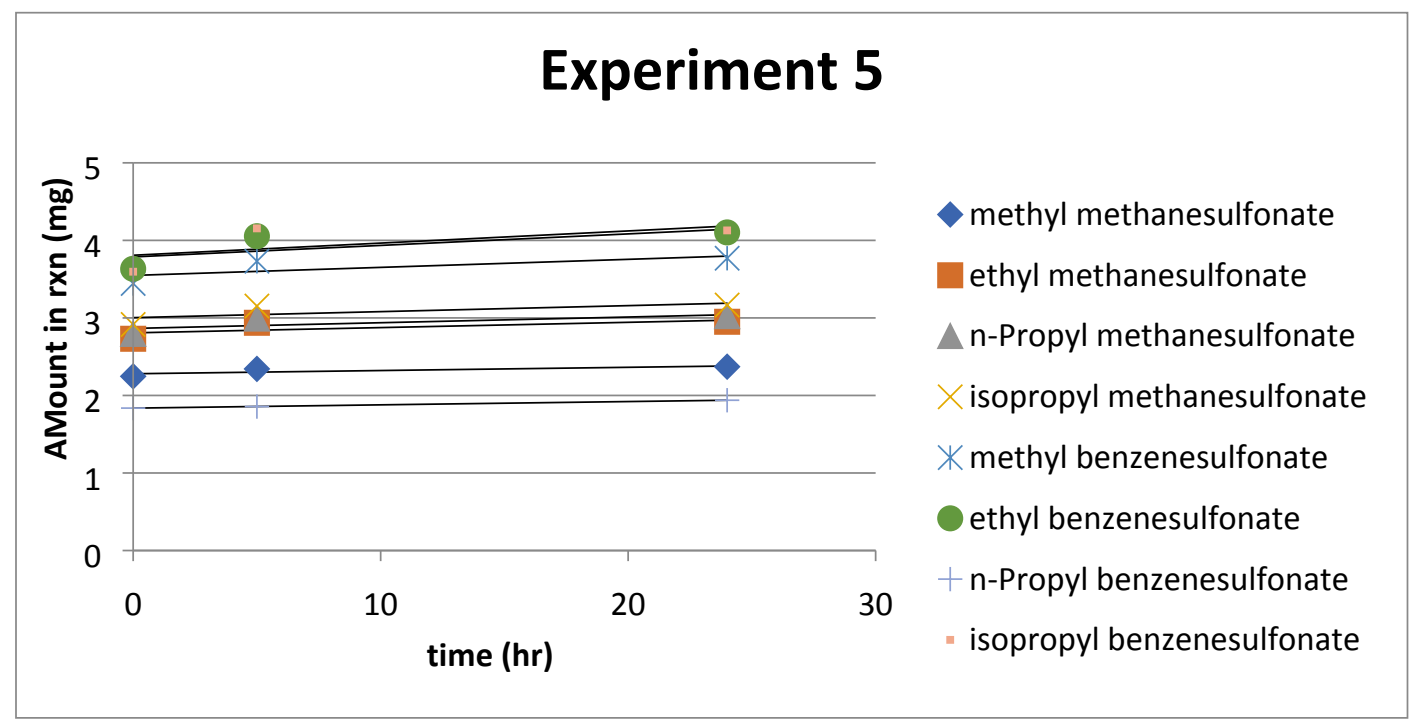

Figure S6. Experiment 6 Stability vs. Time

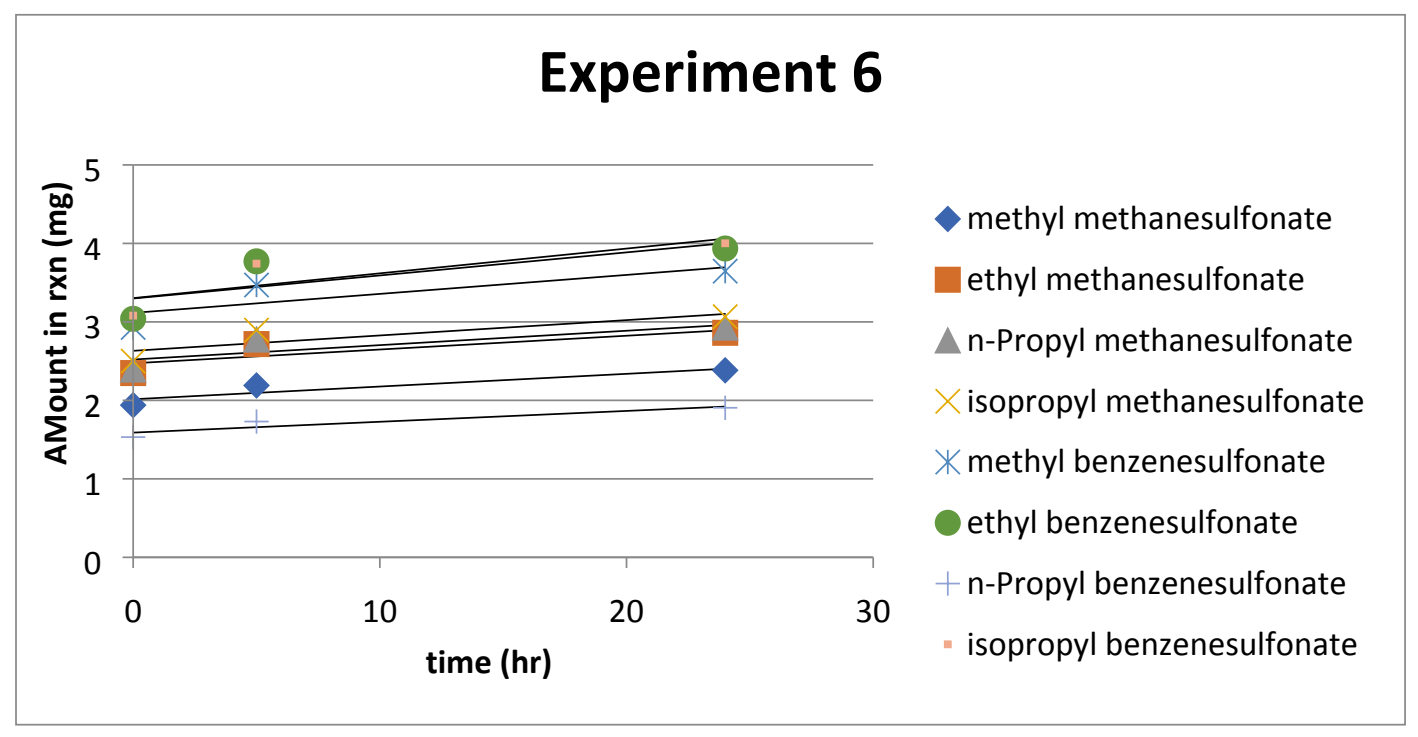

\subsection{Acidic Diluent}

Sample stability was ensured by quenching reactions with acidic diluent ( $80 \%$ acetonitrile, $20 \% 0.1 \mathrm{~N}$ aqueous $\mathrm{HCl}$ ). Data demonstrates that degradation occurs with non-acidic diluent. 
Table S3. Sulfonate Concentration Data With and Without Acidic Diluent Over Time

\begin{tabular}{|c|c|c|}
\hline n-propyl benzenesulfonate & Time (days) & $\begin{array}{c}\text { Concentration in solution } \\
(\mathrm{ng} / \mathrm{mL})\end{array}$ \\
\hline \multirow{3}{*}{$\begin{array}{c}\text { with acid diluent } \\
(80 / 20 \text { acetonitrile/0.1 N HCl })\end{array}$} & 1 & 9829 \\
\cline { 2 - 3 } & 2 & 10087 \\
\hline \multirow{2}{*}{$\begin{array}{c}\text { without acid diluent } \\
(80 / 20 \text { acetonitrile/water })\end{array}$} & 7 & 10871 \\
\cline { 2 - 3 } & 1 & 8736 \\
\cline { 2 - 3 } & 2 & 8353 \\
\hline
\end{tabular}

\subsection{HPLC Internal Standard}

1-phenylnapthelene was added to each stock solution of alkyl sulfonates. Each time point sample was analyzed by both GC/MS and HPLC. GC/MS results were corrected with quantitative HPLC data in order to correct for any sampling error. Graphs below demonstrate the correcting effect of incorporating the HPLC internal standard data.

Figure S7. Example of Scavenger Data Uncorrected with HPLC Internal Standard

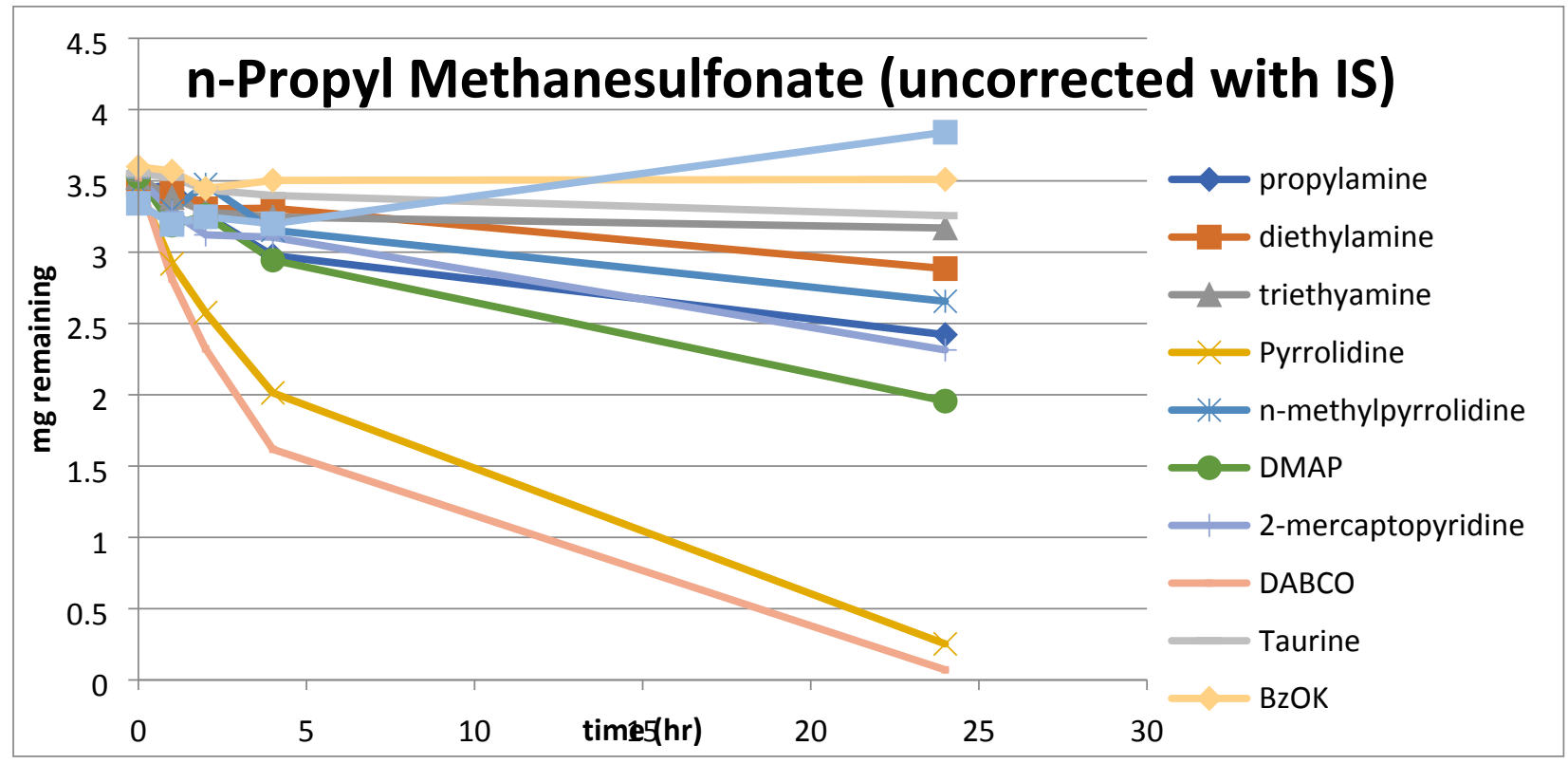


Figure S8. Example of Scavenger Data Corrected with HPLC Internal Standard Correction Factor

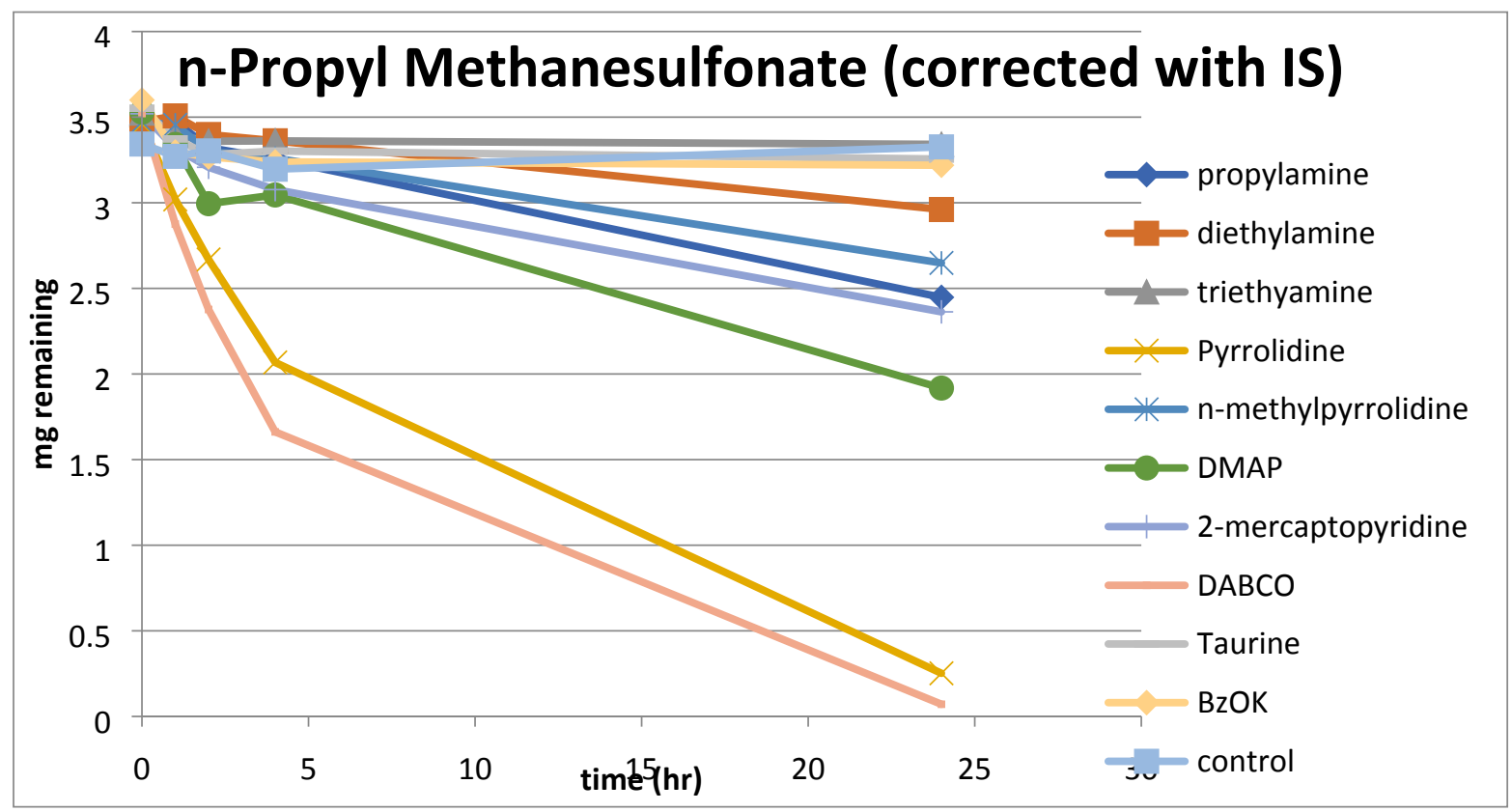

\section{Kinetics Rate Constants:}

Kinetic rate constants were modeled for all data sets collected for each screening experiment in order to understand relative rates of degradation of different sulfonates under different conditions.

Table S4. Modeled Kinetic Rate Constants for Scavenger Data

\begin{tabular}{|c|c|c|c|c|c|c|c|c|c|c|}
\hline Scavenger & Propylamine & diethylamine & triethylamine & pyrrolodine & $\begin{array}{c}\text { n-methyl } \\
\text { pyrrolodine }\end{array}$ & $\begin{array}{c}\text { 4-dimethyl } \\
\text { aminopyridine }\end{array}$ & $\begin{array}{c}\text { 2- } \\
\text { mercapto } \\
\text { pyridine }\end{array}$ & DABCO & taurine & $\begin{array}{c}\text { potassium } \\
\text { benzoate }\end{array}$ \\
\hline EMS & $5.89 \mathrm{E}-04$ & $3.59 \mathrm{E}-04$ & $6.67 \mathrm{E}-05$ & $5.92 \mathrm{E}-03$ & $5.86 \mathrm{E}-04$ & $8.88 \mathrm{E}-04$ & $3.84 \mathrm{E}-04$ & $9.68 \mathrm{E}-03$ & $7.69 \mathrm{E}-05$ & $1.13 \mathrm{E}-04$ \\
\hline nPMS & $2.36 \mathrm{E}-04$ & $1.12 \mathrm{E}-04$ & $4.53 \mathrm{E}-05$ & $2.11 \mathrm{E}-03$ & $1.93 \mathrm{E}-04$ & $4.58 \mathrm{E}-04$ & $3.00 \mathrm{E}-04$ & $3.19 \mathrm{E}-03$ & $7.43 \mathrm{E}-05$ & $9.80 \mathrm{E}-05$ \\
\hline MBS & $6.73 \mathrm{E}-02$ & $1.00 \mathrm{E}+00$ & $2.67 \mathrm{E}-02$ & $6.72 \mathrm{E}-02$ & $1.00 \mathrm{E}+00$ & $1.00 \mathrm{E}+00$ & $2.30 \mathrm{E}-02$ & $1.00 \mathrm{E}+00$ & $1.72 \mathrm{E}-04$ & $3.65 \mathrm{E}-03$ \\
\hline nPBS & 8.97E-04 & 4.09E-04 & $6.49 \mathrm{E}-05$ & $6.91 \mathrm{E}-03$ & $6.80 \mathrm{E}-04$ & $1.34 \mathrm{E}-03$ & $5.83 \mathrm{E}-04$ & $9.80 \mathrm{E}-03$ & $4.26 \mathrm{E}-05$ & $1.11 \mathrm{E}-04$ \\
\hline iPBS & $9.93 \mathrm{E}-05$ & $3.20 \mathrm{E}-05$ & $1.94 \mathrm{E}-12$ & $5.73 \mathrm{E}-04$ & $4.48 \mathrm{E}-05$ & $1.28 \mathrm{E}-04$ & $4.85 \mathrm{E}-10$ & $8.48 \mathrm{E}-04$ & $6.09 \mathrm{E}-12$ & $5.84 \mathrm{E}-05$ \\
\hline
\end{tabular}

Table S5. Modeled Kinetic Rate Constants for Solvents + DABCO

\begin{tabular}{|c|c|c|c|c|c|c|c|c|}
\hline \multirow{3}{*}{ Solvent } & $\begin{array}{c}2 \text { MeTHF } \\
+ \\
\text { DABCO }\end{array}$ & $\begin{array}{c}\text { THF }+ \\
\text { DABCO }\end{array}$ & $\begin{array}{c}\text { NMP }+ \\
\text { DABCO }\end{array}$ & $\begin{array}{c}\text { ACN }+ \\
\text { DABCO }\end{array}$ & $\begin{array}{c}\text { EtOH }+ \\
\text { DABCO }\end{array}$ & $\begin{array}{c}\text { EtOAc }+ \\
\text { DABCO }\end{array}$ & $\begin{array}{c}\text { MTBE }+ \\
\text { DABCO }\end{array}$ & $\begin{array}{c}\text { toleune }+ \\
\text { DABCO }\end{array}$ \\
\hline
\end{tabular}




\begin{tabular}{|c|c|c|c|c|c|c|c|c|}
\hline MMS & $1.00 \mathrm{E}+00$ & $1.00 \mathrm{E}+00$ & $1.00 \mathrm{E}+00$ & $1.00 \mathrm{E}+00$ & $1.00 \mathrm{E}+00$ & $1.00 \mathrm{E}+00$ & $1.00 \mathrm{E}+00$ & $1.00 \mathrm{E}+00$ \\
\hline EMS & $9.10 \mathrm{E}-03$ & $1.38 \mathrm{E}-02$ & $6.99 \mathrm{E}-02$ & $1.35 \mathrm{E}-01$ & $3.76 \mathrm{E}-02$ & $2.47 \mathrm{E}-02$ & $6.30 \mathrm{E}-03$ & $1.74 \mathrm{E}-02$ \\
\hline $\mathbf{n P M S}$ & $2.43 \mathrm{E}-03$ & $3.99 \mathrm{E}-03$ & $2.97 \mathrm{E}-02$ & $4.82 \mathrm{E}-02$ & $1.34 \mathrm{E}-02$ & $9.87 \mathrm{E}-03$ & $1.55 \mathrm{E}-03$ & $4.81 \mathrm{E}-03$ \\
\hline iPMS & $1.57 \mathrm{E}-04$ & $2.38 \mathrm{E}-04$ & $2.23 \mathrm{E}-03$ & $4.20 \mathrm{E}-03$ & $2.36 \mathrm{E}-03$ & $2.62 \mathrm{E}-03$ & $1.61 \mathrm{E}-04$ & $3.08 \mathrm{E}-04$ \\
\hline MBS & $1.00 \mathrm{E}+00$ & $1.00 \mathrm{E}+00$ & $1.00 \mathrm{E}+00$ & $1.00 \mathrm{E}+00$ & $1.00 \mathrm{E}+00$ & $1.00 \mathrm{E}+00$ & $1.00 \mathrm{E}+00$ & $1.00 \mathrm{E}+00$ \\
\hline nPBS & $1.00 \mathrm{E}-02$ & $1.70 \mathrm{E}-02$ & $6.33 \mathrm{E}-02$ & $6.00 \mathrm{E}-02$ & $2.68 \mathrm{E}-02$ & $1.43 \mathrm{E}-02$ & $3.27 \mathrm{E}-03$ & $1.13 \mathrm{E}-02$ \\
\hline iPBS & $4.22 \mathrm{E}-04$ & $7.91 \mathrm{E}-04$ & $2.28 \mathrm{E}-02$ & $2.89 \mathrm{E}-02$ & $4.30 \mathrm{E}-03$ & $3.21 \mathrm{E}-03$ & $2.53 \mathrm{E}-04$ & $5.54 \mathrm{E}-04$ \\
\hline
\end{tabular}

Table S6. Modeled Kinetic Rate Constants for Solvents + DABCO + Water:

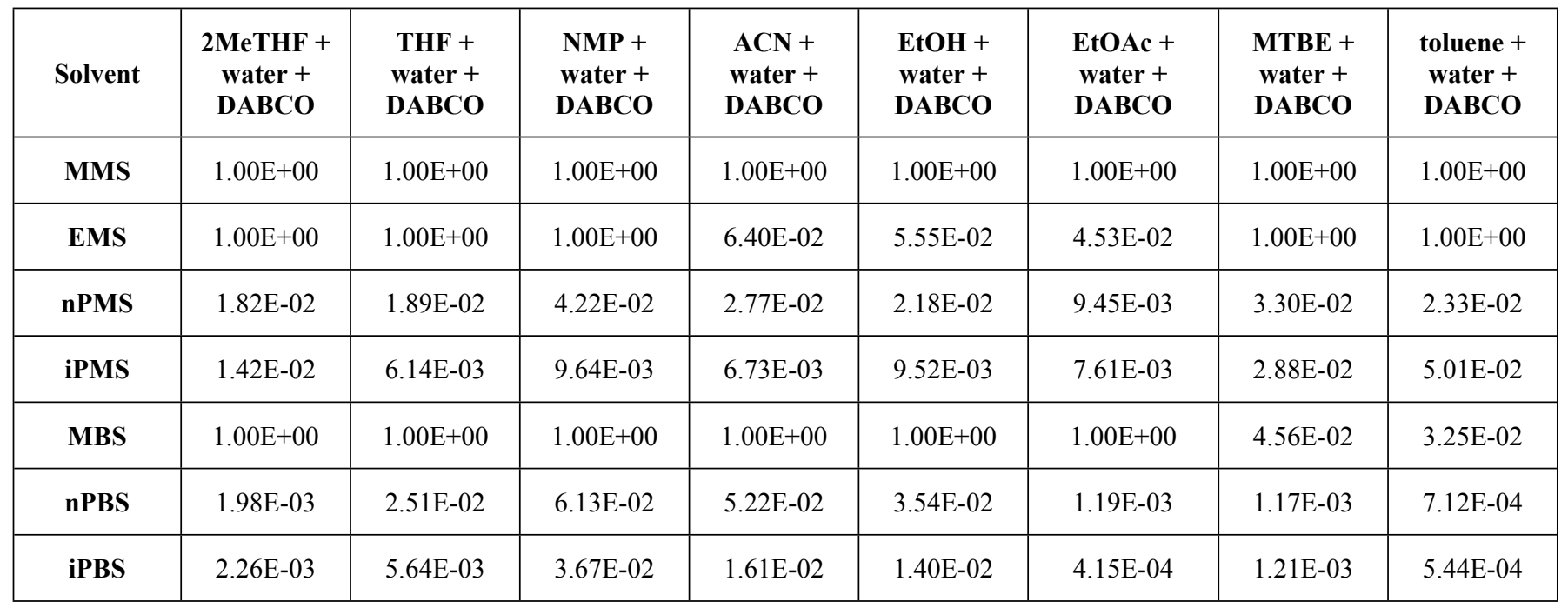

Table S7. Modeled Kinetic Rate Constants for Solvents + Water:

\begin{tabular}{|c|c|c|c|c|c|c|c|c|}
\hline Solvent & $\begin{array}{c}\text { 2MeTHF + } \\
\text { water }\end{array}$ & $\begin{array}{l}\text { THF + } \\
\text { water }\end{array}$ & $\begin{array}{c}\text { NMP + } \\
\text { water }\end{array}$ & $\begin{array}{c}\mathrm{ACN}+ \\
\text { water }\end{array}$ & $\begin{array}{c}\text { EtOH + } \\
\text { water }\end{array}$ & $\begin{array}{c}\text { EtOAc + } \\
\text { water }\end{array}$ & $\begin{array}{l}\text { MTBE + } \\
\text { water }\end{array}$ & $\begin{array}{c}\text { toluene }+ \\
\text { water }\end{array}$ \\
\hline MMS & $1.57 \mathrm{E}-03$ & $6.16 \mathrm{E}-04$ & $1.17 \mathrm{E}-03$ & $2.51 \mathrm{E}-04$ & $1.38 \mathrm{E}-03$ & $7.02 \mathrm{E}-04$ & $4.80 \mathrm{E}-03$ & $4.86 \mathrm{E}-03$ \\
\hline EMS & 4.82E-04 & $2.41 \mathrm{E}-04$ & $3.67 \mathrm{E}-04$ & $1.08 \mathrm{E}-04$ & $5.83 \mathrm{E}-04$ & $2.72 \mathrm{E}-04$ & $1.17 \mathrm{E}-03$ & $9.97 \mathrm{E}-04$ \\
\hline nPMS & $1.46 \mathrm{E}-04$ & $1.35 \mathrm{E}-04$ & $2.30 \mathrm{E}-04$ & 7.17E-05 & $3.29 \mathrm{E}-04$ & $9.88 \mathrm{E}-05$ & $2.41 \mathrm{E}-04$ & $2.18 \mathrm{E}-04$ \\
\hline iPMS & 7.05E-03 & $1.66 \mathrm{E}-03$ & $1.51 \mathrm{E}-03$ & $1.02 \mathrm{E}-03$ & $4.18 \mathrm{E}-03$ & $4.34 \mathrm{E}-03$ & $1.69 \mathrm{E}-02$ & $1.31 \mathrm{E}-02$ \\
\hline MBS & $1.76 \mathrm{E}-04$ & 8.53E-04 & $4.52 \mathrm{E}-03$ & $5.21 \mathrm{E}-04$ & $2.41 \mathrm{E}-03$ & $1.19 \mathrm{E}-04$ & $1.31 \mathrm{E}-04$ & $1.18 \mathrm{E}-04$ \\
\hline nPBS & 7.32E-05 & $2.31 \mathrm{E}-04$ & $9.50 \mathrm{E}-04$ & $1.96 \mathrm{E}-04$ & $7.15 \mathrm{E}-04$ & $8.64 \mathrm{E}-05$ & $3.84 \mathrm{E}-05$ & $5.81 \mathrm{E}-05$ \\
\hline iPBS & $5.56 \mathrm{E}-04$ & $2.10 \mathrm{E}-03$ & $6.28 \mathrm{E}-03$ & $2.92 \mathrm{E}-03$ & 7.14E-03 & $4.58 \mathrm{E}-04$ & $7.25 \mathrm{E}-04$ & $3.68 \mathrm{E}-04$ \\
\hline
\end{tabular}




\section{3. \% Removal Data}

Table S8. \% Removal Data: Scavengers

\begin{tabular}{|c|c|c|c|c|}
\hline$\%$ removal & $1 \mathrm{hr}$ & $2 \mathrm{hr}$ & $4 \mathrm{hr}$ & $24 \mathrm{~h}$ \\
\hline \multicolumn{5}{|c|}{ propylamine } \\
\hline methyl methanesulfonate & 90 & 98 & 99 & 99 \\
\hline ethyl methanesulfonate & 2 & 8 & 14 & 57 \\
\hline n-Propyl methanesulfonate & 0 & 4 & 5 & 29 \\
\hline isopropyl methanesulfonate & 0 & 1 & 1 & 3 \\
\hline methyl benzenesulfonate & 98 & 100 & 100 & 100 \\
\hline ethyl benzenesulfonate & 16 & 29 & 44 & 93 \\
\hline n-Propyl benzenesulfonate & 6 & 14 & 23 & 70 \\
\hline isopropyl benzenesulfonate & 0 & 0 & 5 & 13 \\
\hline
\end{tabular}

\begin{tabular}{|l|r|r|r|r|}
\hline \multicolumn{5}{|c|}{ diethylamine } \\
\hline methyl methanesulfonate & 93 & 99 & 99 & 99 \\
\hline ethyl methanesulfonate & 2 & 7 & 10 & 40 \\
\hline n-Propyl methanesulfonate & 0 & 2 & 3 & 15 \\
\hline isopropyl methanesulfonate & 0 & 1 & 2 & 3 \\
\hline methyl benzenesulfonate & 100 & 100 & 100 & 100 \\
\hline ethyl benzenesulfonate & 10 & 19 & 31 & 83 \\
\hline n-Propyl benzenesulfonate & 0 & 8 & 10 & 44 \\
\hline isopropyl benzenesulfonate & 0 & 0 & 4 & 4
\end{tabular}

\begin{tabular}{|l|r|r|r|r|}
\hline \multicolumn{5}{|c|}{ triethylamine } \\
\hline methyl methanesulfonate & 38 & 58 & 81 & 99 \\
\hline ethyl methanesulfonate & 3 & 5 & 5 & 8 \\
\hline n-Propyl methanesulfonate & 4 & 5 & 5 & 5 \\
\hline isopropyl methanesulfonate & 3 & 3 & 4 & 2 \\
\hline methyl benzenesulfonate & 81 & 94 & 98 & 100 \\
\hline ethyl benzenesulfonate & 8 & 11 & 11 & 21 \\
\hline n-Propyl benzenesulfonate & 4 & 6 & 8 & 7 \\
\hline isopropyl benzenesulfonate & 0 & 1 & 3 & 0
\end{tabular}

\begin{tabular}{|l|r|r|r|r|}
\hline \multicolumn{5}{|c|}{ Pyrrolidine } \\
\hline methyl methanesulfonate & 100 & 99 & 100 & 99 \\
\hline
\end{tabular}




\begin{tabular}{|l|r|r|r|r|}
\hline ethyl methanesulfonate & 31 & 51 & 75 & 99 \\
\hline n-Propyl methanesulfonate & 13 & 23 & 40 & 93 \\
\hline isopropyl methanesulfonate & 2 & 4 & 7 & 22 \\
\hline methyl benzenesulfonate & 98 & 100 & 98 & 98 \\
\hline ethyl benzenesulfonate & 72 & 90 & 97 & 95 \\
\hline n-Propyl benzenesulfonate & 34 & 56 & 82 & 98 \\
\hline isopropyl benzenesulfonate & 0 & 2 & 15 & 57
\end{tabular}

\begin{tabular}{|l|r|r|r|r|}
\hline \multicolumn{5}{|c|}{ n-methylpyrrolidine } \\
\hline methyl methanesulfonate & 98 & 99 & 100 & 100 \\
\hline ethyl methanesulfonate & 3 & 9 & 15 & 56 \\
\hline n-Propyl methanesulfonate & 0 & 5 & 6 & 24 \\
\hline isopropyl methanesulfonate & 0 & 2 & 2 & 3 \\
\hline methyl benzenesulfonate & 100 & 98 & 100 & 100 \\
\hline ethyl benzenesulfonate & 15 & 29 & 45 & 94 \\
\hline n-Propyl benzenesulfonate & 4 & 10 & 16 & 62 \\
\hline isopropyl benzenesulfonate & 0 & 0 & 0 & 7
\end{tabular}

\begin{tabular}{|l|r|r|r|r|}
\hline \multicolumn{7}{|c|}{ DMAP } \\
\hline methyl methanesulfonate & 89 & 98 & 96 & 96 \\
\hline ethyl methanesulfonate & 8 & 19 & 21 & 68 \\
\hline n-Propyl methanesulfonate & 5 & 15 & 14 & 46 \\
\hline isopropyl methanesulfonate & 4 & 12 & 7 & 9 \\
\hline methyl benzenesulfonate & 98 & 100 & 98 & 98 \\
\hline ethyl benzenesulfonate & 13 & 32 & 50 & 97 \\
\hline n-Propyl benzenesulfonate & 3 & 18 & 26 & 86 \\
\hline isopropyl benzenesulfonate & 0 & 0 & 0 & 12
\end{tabular}

\begin{tabular}{|l|r|r|r|r|}
\hline \multicolumn{5}{|c|}{ 2-mercaptopyridine } \\
\hline methyl methanesulfonate & 41 & 62 & 85 & 99 \\
\hline ethyl methanesulfonate & 6 & 10 & 14 & 40 \\
\hline n-Propyl methanesulfonate & 6 & 9 & 13 & 33 \\
\hline isopropyl methanesulfonate & 5 & 7 & 9 & 9 \\
\hline methyl benzenesulfonate & 76 & 92 & 98 & 100 \\
\hline ethyl benzenesulfonate & 2 & 11 & 19 & 71 \\
\hline n-Propyl benzenesulfonate & 0 & 2 & 9 & 59 \\
\hline isopropyl benzenesulfonate & 0 & 0 & 0 & 0
\end{tabular}




\begin{tabular}{|l|r|r|r|r|}
\hline \multicolumn{7}{|c|}{ DABCO } \\
\hline methyl methanesulfonate & 100 & 99 & 99 & 99 \\
\hline ethyl methanesulfonate & 44 & 68 & 90 & 100 \\
\hline n-Propyl methanesulfonate & 18 & 32 & 53 & 98 \\
\hline isopropyl methanesulfonate & 4 & 8 & 10 & 33 \\
\hline methyl benzenesulfonate & 100 & 100 & 98 & 100 \\
\hline ethyl benzenesulfonate & 85 & 96 & 92 & 96 \\
\hline n-Propyl benzenesulfonate & 42 & 71 & 91 & 98 \\
\hline isopropyl benzenesulfonate & 0 & 3 & 17 & 74
\end{tabular}

\begin{tabular}{|l|r|r|r|r|}
\hline \multicolumn{5}{|c|}{ Taurine } \\
\hline methyl methanesulfonate & 6 & 11 & 13 & 16 \\
\hline ethyl methanesulfonate & 5 & 8 & 7 & 9 \\
\hline n-Propyl methanesulfonate & 5 & 8 & 7 & 8 \\
\hline isopropyl methanesulfonate & 5 & 8 & 8 & 7 \\
\hline methyl benzenesulfonate & 7 & 17 & 19 & 17 \\
\hline ethyl benzenesulfonate & 3 & 8 & 6 & 7 \\
\hline n-Propyl benzenesulfonate & 3 & 8 & 8 & 4 \\
\hline isopropyl benzenesulfonate & 0 & 6 & 6 & 0
\end{tabular}

\begin{tabular}{|l|r|r|r|r|}
\hline \multicolumn{5}{|c|}{$\mathrm{NaOBz}$} \\
\hline methyl methanesulfonate & 15 & 21 & 28 & 47 \\
\hline ethyl methanesulfonate & 9 & 9 & 10 & 12 \\
\hline n-Propyl methanesulfonate & 8 & 9 & 10 & 11 \\
\hline isopropyl methanesulfonate & 8 & 9 & 10 & 8 \\
\hline methyl benzenesulfonate & 27 & 40 & 53 & 75 \\
\hline ethyl benzenesulfonate & 21 & 12 & 14 & 31 \\
\hline n-Propyl benzenesulfonate & 9 & 11 & 11 & 12 \\
\hline isopropyl benzenesulfonate & 8 & 10 & 13 & 5
\end{tabular}

\begin{tabular}{|l|r|r|r|r|}
\hline \multicolumn{5}{|c|}{ control (no reagent) } \\
\hline methyl methanesulfonate & 1 & 2 & 7 & 0 \\
\hline ethyl methanesulfonate & 1 & 1 & 4 & 0 \\
\hline n-Propyl methanesulfonate & 2 & 1 & 4 & 1 \\
\hline isopropyl methanesulfonate & 2 & 2 & 5 & 0 \\
\hline methyl benzenesulfonate & 7 & 9 & 14 & 2 \\
\hline ethyl benzenesulfonate & 6 & 4 & 8 & 1 \\
\hline n-Propyl benzenesulfonate & 8 & 6 & 6 & 0 \\
\hline
\end{tabular}




\begin{tabular}{|l|l|l|l|l|}
\hline isopropyl benzenesulfonate & 7 & 8 & 11 & 1 \\
\hline
\end{tabular}

Table 9. \% Removal Data: Solvent

\begin{tabular}{|l|r|r|r|r|}
\hline \% removal & \multicolumn{5}{|l|}{$1 \mathrm{hr}$} & \multicolumn{1}{l|}{ 2hr } & 4hr & \multicolumn{1}{l|}{$24 \mathrm{hr}$} \\
\hline \multicolumn{5}{|c|}{ 2MeTHF + DABCO } \\
\hline methyl methanesulfonate & 100 & 100 & 98 & 98 \\
\hline ethyl methanesulfonate & 44 & 66 & 88 & 100 \\
\hline n-Propyl methanesulfonate & 14 & 24 & 45 & 96 \\
\hline isopropyl methanesulfonate & 0 & 0 & 0 & 23 \\
\hline methyl benzenesulfonate & 100 & 100 & 100 & 100 \\
\hline n-Propyl benzenesulfonate & 43 & 66 & 88 & 100 \\
\hline isopropyl benzenesulfonate & 0 & 0 & 0 & 61
\end{tabular}

\begin{tabular}{|l|r|r|r|r|}
\hline \multicolumn{5}{|c|}{ THF + DABCO } \\
\hline methyl methanesulfonate & 100 & 98 & 98 & 100 \\
\hline ethyl methanesulfonate & 58 & 80 & 95 & 100 \\
\hline n-Propyl methanesulfonate & 21 & 39 & 61 & 98 \\
\hline isopropyl methanesulfonate & 0 & 1 & 0 & 31 \\
\hline methyl benzenesulfonate & 97 & 100 & 100 & 100 \\
\hline n-Propyl benzenesulfonate & 65 & 86 & 96 & 97 \\
\hline isopropyl benzenesulfonate & 0 & 0 & 3 & 83
\end{tabular}

\begin{tabular}{|l|r|r|r|r|}
\hline \multicolumn{5}{|c|}{ NMP + DABCO } \\
\hline methyl methanesulfonate & 98 & 99 & 99 & 100 \\
\hline ethyl methanesulfonate & 99 & 100 & 100 & 100 \\
\hline n-Propyl methanesulfonate & 84 & 95 & 98 & 98 \\
\hline isopropyl methanesulfonate & 12 & 20 & 44 & 96 \\
\hline methyl benzenesulfonate & 100 & 100 & 100 & 100 \\
\hline n-Propyl benzenesulfonate & 98 & 100 & 98 & 98 \\
\hline isopropyl benzenesulfonate & 76 & 91 & 97 & 98
\end{tabular}

\begin{tabular}{|l|r|r|r|r|}
\hline \multicolumn{5}{|c|}{ MeCN + DABCO } \\
\hline methyl methanesulfonate & 98 & 98 & 100 & 98 \\
\hline ethyl methanesulfonate & 100 & 100 & 100 & 99 \\
\hline
\end{tabular}




\begin{tabular}{|l|r|r|r|r|}
\hline n-Propyl methanesulfonate & 95 & 98 & 98 & 100 \\
\hline isopropyl methanesulfonate & 21 & 39 & 65 & 98 \\
\hline methyl benzenesulfonate & 100 & 97 & 100 & 100 \\
\hline n-Propyl benzenesulfonate & 97 & 100 & 100 & 100 \\
\hline isopropyl benzenesulfonate & 83 & 94 & 97 & 100
\end{tabular}

\begin{tabular}{|l|r|r|r|r|}
\hline \multicolumn{5}{|c|}{ EtOH + DABCO } \\
\hline methyl methanesulfonate & 98 & 100 & 100 & 98 \\
\hline ethyl methanesulfonate & 90 & 97 & 98 & 100 \\
\hline n-Propyl methanesulfonate & 57 & 79 & 95 & 100 \\
\hline isopropyl methanesulfonate & 12 & 23 & 45 & 96 \\
\hline methyl benzenesulfonate & 100 & 100 & 97 & 100 \\
\hline n-Propyl benzenesulfonate & 81 & 93 & 97 & 100 \\
\hline isopropyl benzenesulfonate & 12 & 38 & 72 & 96
\end{tabular}

\begin{tabular}{|l|r|r|r|r|}
\hline \multicolumn{5}{|c|}{ EtOAc + DABCO } \\
\hline methyl methanesulfonate & 99 & 100 & 97 & 99 \\
\hline ethyl methanesulfonate & 79 & 91 & 98 & 100 \\
\hline n-Propyl methanesulfonate & 55 & 66 & 81 & 99 \\
\hline isopropyl methanesulfonate & 36 & 37 & 40 & 62 \\
\hline methyl benzenesulfonate & 100 & 100 & 100 & 100 \\
\hline n-Propyl benzenesulfonate & 47 & 94 & 98 & 98 \\
\hline isopropyl benzenesulfonate & 19 & 37 & 50 & 93
\end{tabular}

\begin{tabular}{|l|r|r|r|r|}
\hline \multicolumn{5}{|c|}{ MTBE + DABCO } \\
\hline methyl methanesulfonate & 98 & 98 & 100 & 98 \\
\hline ethyl methanesulfonate & 31 & 52 & 79 & 100 \\
\hline n-Propyl methanesulfonate & 4 & 13 & 32 & 92 \\
\hline isopropyl methanesulfonate & 0 & 0 & 0 & 23 \\
\hline methyl benzenesulfonate & 100 & 100 & 100 & 100 \\
\hline n-Propyl benzenesulfonate & 12 & 30 & 59 & 97 \\
\hline isopropyl benzenesulfonate & 0 & 0 & 0 & 43
\end{tabular}

\begin{tabular}{|l|r|r|r|r|}
\hline \multicolumn{5}{|c|}{ toluene + DABCO } \\
\hline methyl methanesulfonate & 100 & 98 & 98 & 100 \\
\hline ethyl methanesulfonate & 67 & 85 & 97 & 100 \\
\hline n-Propyl methanesulfonate & 26 & 43 & 69 & 98 \\
\hline
\end{tabular}




\begin{tabular}{|l|r|r|r|r|}
\hline isopropyl methanesulfonate & 0 & 0 & 4 & 38 \\
\hline methyl benzenesulfonate & 100 & 100 & 100 & 100 \\
\hline n-Propyl benzenesulfonate & 51 & 73 & 91 & 100 \\
\hline isopropyl benzenesulfonate & 0 & 0 & 0 & 69
\end{tabular}

\begin{tabular}{|l|r|r|r|r|}
\hline \multicolumn{5}{|c|}{ 2MeTHF + DABCO + water } \\
\hline methyl methanesulfonate & 98 & 100 & 99 & 100 \\
\hline ethyl methanesulfonate & 98 & 98 & 100 & 99 \\
\hline n-Propyl methanesulfonate & 66 & 90 & 99 & 99 \\
\hline isopropyl methanesulfonate & 56 & 83 & 98 & 99 \\
\hline methyl benzenesulfonate & 98 & 100 & 99 & 98 \\
\hline n-Propyl benzenesulfonate & 4 & 18 & 41 & 96 \\
\hline isopropyl benzenesulfonate & 0 & 5 & 62 & 91
\end{tabular}

\begin{tabular}{|l|r|r|r|r|}
\hline \multicolumn{5}{|c|}{ THF + DABCO + water } \\
\hline methyl methanesulfonate & 98 & 98 & 98 & 98 \\
\hline ethyl methanesulfonate & 97 & 100 & 100 & 100 \\
\hline n-Propyl methanesulfonate & 69 & 89 & 98 & 98 \\
\hline isopropyl methanesulfonate & 31 & 51 & 78 & 98 \\
\hline methyl benzenesulfonate & 97 & 100 & 100 & 100 \\
\hline n-Propyl benzenesulfonate & 79 & 93 & 97 & 95 \\
\hline isopropyl benzenesulfonate & 21 & 49 & 79 & 100
\end{tabular}

\begin{tabular}{|l|r|r|r|r|}
\hline \multicolumn{5}{|c|}{ NMP + DABCO + water } \\
\hline methyl methanesulfonate & 99 & 98 & 99 & 100 \\
\hline ethyl methanesulfonate & 99 & 100 & 100 & 100 \\
\hline n-Propyl methanesulfonate & 92 & 98 & 99 & 98 \\
\hline isopropyl methanesulfonate & 45 & 68 & 90 & 99 \\
\hline methyl benzenesulfonate & 100 & 100 & 100 & 98 \\
\hline n-Propyl benzenesulfonate & 97 & 100 & 98 & 100 \\
\hline isopropyl benzenesulfonate & 89 & 96 & 98 & 98
\end{tabular}

\begin{tabular}{|l|r|r|r|r|}
\hline \multicolumn{5}{|c|}{ MeCN + DABCO + water } \\
\hline methyl methanesulfonate & 98 & 98 & 100 & 100 \\
\hline ethyl methanesulfonate & 98 & 100 & 99 & 100 \\
\hline n-Propyl methanesulfonate & 82 & 95 & 99 & 99 \\
\hline
\end{tabular}




\begin{tabular}{|l|r|r|r|r|}
\hline isopropyl methanesulfonate & 34 & 55 & 80 & 99 \\
\hline methyl benzenesulfonate & 100 & 100 & 100 & 100 \\
\hline n-Propyl benzenesulfonate & 96 & 97 & 97 & 97 \\
\hline isopropyl benzenesulfonate & 62 & 86 & 96 & 97
\end{tabular}

\begin{tabular}{|l|r|r|r|r|}
\hline \multicolumn{5}{|c|}{ EtOH + DABCO + water } \\
\hline methyl methanesulfonate & 98 & 100 & 100 & 100 \\
\hline ethyl methanesulfonate & 97 & 98 & 98 & 98 \\
\hline n-Propyl methanesulfonate & 74 & 91 & 98 & 98 \\
\hline isopropyl methanesulfonate & 44 & 68 & 89 & 98 \\
\hline methyl benzenesulfonate & 100 & 100 & 97 & 100 \\
\hline n-Propyl benzenesulfonate & 89 & 96 & 100 & 96 \\
\hline isopropyl benzenesulfonate & 57 & 82 & 94 & 96
\end{tabular}

\begin{tabular}{|l|r|r|r|r|}
\hline \multicolumn{5}{|c|}{ EtOAc + DABCO + water } \\
\hline methyl methanesulfonate & 98 & 98 & 100 & 100 \\
\hline ethyl methanesulfonate & 94 & 98 & 98 & 100 \\
\hline n-Propyl methanesulfonate & 47 & 66 & 88 & 99 \\
\hline isopropyl methanesulfonate & 40 & 58 & 83 & 99 \\
\hline methyl benzenesulfonate & 98 & 97 & 97 & 100 \\
\hline n-Propyl benzenesulfonate & 5 & 9 & 29 & 81 \\
\hline isopropyl benzenesulfonate & 0 & 0 & 0 & 54
\end{tabular}

\begin{tabular}{|l|r|r|r|r|}
\hline \multicolumn{5}{|c|}{ MTBE + DABCO + water } \\
\hline methyl methanesulfonate & 98 & 100 & 100 & 100 \\
\hline ethyl methanesulfonate & 100 & 100 & 100 & 100 \\
\hline n-Propyl methanesulfonate & 87 & 97 & 98 & 100 \\
\hline isopropyl methanesulfonate & 83 & 96 & 98 & 99 \\
\hline methyl benzenesulfonate & 94 & 97 & 100 & 100 \\
\hline n-Propyl benzenesulfonate & 0 & 2 & 24 & 88 \\
\hline isopropyl benzenesulfonate & 0 & 15 & 21 & 89
\end{tabular}

\begin{tabular}{|l|r|r|r|r|}
\hline \multicolumn{5}{|c|}{ toluene + DABCO + water } \\
\hline methyl methanesulfonate & 100 & 100 & 100 & 100 \\
\hline ethyl methanesulfonate & 98 & 100 & 100 & 100 \\
\hline n-Propyl methanesulfonate & 76 & 93 & 98 & 100 \\
\hline isopropyl methanesulfonate & 96 & 93 & 98 & 99 \\
\hline
\end{tabular}




\begin{tabular}{|l|r|r|r|r|}
\hline methyl benzenesulfonate & 85 & 100 & 98 & 100 \\
\hline n-Propyl benzenesulfonate & 0 & 0 & 7 & 72 \\
\hline isopropyl benzenesulfonate & 0 & 0 & 0 & 67 \\
\hline
\end{tabular}

\begin{tabular}{|l|r|r|r|r|}
\hline \multicolumn{5}{|c|}{ 2MeTHF + water } \\
\hline methyl methanesulfonate & 17 & 24 & 33 & 80 \\
\hline ethyl methanesulfonate & 8 & 13 & 17 & 47 \\
\hline n-Propyl methanesulfonate & 3 & 7 & 9 & 17 \\
\hline isopropyl methanesulfonate & 35 & 57 & 81 & 99 \\
\hline methyl benzenesulfonate & 2 & 6 & 9 & 21 \\
\hline n-Propyl benzenesulfonate & 2 & 5 & 7 & 8 \\
\hline isopropyl benzenesulfonate & 6 & 12 & 18 & 52
\end{tabular}

\begin{tabular}{|l|r|r|r|r|}
\hline \multicolumn{5}{|c|}{ THF + water } \\
\hline methyl methanesulfonate & 8 & 14 & 11 & 58 \\
\hline ethyl methanesulfonate & 6 & 8 & 2 & 29 \\
\hline n-Propyl methanesulfonate & 5 & 8 & 0 & 18 \\
\hline isopropyl methanesulfonate & 13 & 23 & 29 & 90 \\
\hline methyl benzenesulfonate & 9 & 15 & 15 & 70 \\
\hline n-Propyl benzenesulfonate & 6 & 9 & 3 & 28 \\
\hline isopropyl benzenesulfonate & 16 & 27 & 36 & 92
\end{tabular}

\begin{tabular}{|l|r|r|r|r|}
\hline \multicolumn{5}{|c|}{ NMP + water } \\
\hline methyl methanesulfonate & 8 & 17 & 27 & 78 \\
\hline ethyl methanesulfonate & 3 & 8 & 12 & 40 \\
\hline n-Propyl methanesulfonate & 3 & 7 & 9 & 27 \\
\hline isopropyl methanesulfonate & 10 & 20 & 33 & 83 \\
\hline methyl benzenesulfonate & 24 & 42 & 65 & 98 \\
\hline n-Propyl benzenesulfonate & 7 & 14 & 24 & 72 \\
\hline isopropyl benzenesulfonate & 32 & 54 & 76 & 98
\end{tabular}

\begin{tabular}{|l|r|r|r|r|}
\hline \multicolumn{5}{|c|}{$\mathrm{MeCN}+$ water } \\
\hline methyl methanesulfonate & 5 & 8 & 10 & 29 \\
\hline ethyl methanesulfonate & 3 & 5 & 5 & 13 \\
\hline n-Propyl methanesulfonate & 3 & 5 & 4 & 9 \\
\hline isopropyl methanesulfonate & 9 & 15 & 24 & 74 \\
\hline
\end{tabular}




\begin{tabular}{|l|r|r|r|r|}
\hline methyl benzenesulfonate & 7 & 10 & 15 & 51 \\
\hline n-Propyl benzenesulfonate & 3 & 5 & 8 & 23 \\
\hline isopropyl benzenesulfonate & 18 & 31 & 49 & 95 \\
\hline
\end{tabular}

\begin{tabular}{|l|r|r|r|r|}
\hline \multicolumn{5}{|c|}{ EtOH + water } \\
\hline methyl methanesulfonate & 9 & 17 & 30 & 84 \\
\hline ethyl methanesulfonate & 10 & 8 & 14 & 56 \\
\hline n-Propyl methanesulfonate & 11 & 5 & 9 & 37 \\
\hline isopropyl methanesulfonate & 13 & 42 & 66 & 98 \\
\hline methyl benzenesulfonate & 11 & 26 & 45 & 95 \\
\hline n-Propyl benzenesulfonate & 12 & 10 & 20 & 61 \\
\hline isopropyl benzenesulfonate & 23 & 63 & 86 & 100
\end{tabular}

\begin{tabular}{|l|r|r|r|r|}
\hline \multicolumn{5}{|c|}{ EtOAc + water } \\
\hline methyl methanesulfonate & 12 & 19 & 21 & 58 \\
\hline ethyl methanesulfonate & 7 & 13 & 11 & 30 \\
\hline n-Propyl methanesulfonate & 5 & 10 & 7 & 11 \\
\hline isopropyl methanesulfonate & 25 & 43 & 62 & 99 \\
\hline methyl benzenesulfonate & 5 & 10 & 7 & 14 \\
\hline n-Propyl benzenesulfonate & 4 & 9 & 7 & 10 \\
\hline isopropyl benzenesulfonate & 8 & 14 & 16 & 45
\end{tabular}

\begin{tabular}{|l|r|r|r|r|}
\hline \multicolumn{5}{|c|}{ MTBE + water } \\
\hline methyl methanesulfonate & 39 & 46 & 58 & 95 \\
\hline ethyl methanesulfonate & 18 & 22 & 29 & 73 \\
\hline n-Propyl methanesulfonate & 6 & 6 & 8 & 28 \\
\hline isopropyl methanesulfonate & 66 & 84 & 97 & 99 \\
\hline methyl benzenesulfonate & 2 & 2 & 4 & 17 \\
\hline n-Propyl benzenesulfonate & 2 & 0 & 1 & 5 \\
\hline isopropyl benzenesulfonate & 7 & 10 & 17 & 64
\end{tabular}

\begin{tabular}{|l|r|r|r|r|}
\hline \multicolumn{5}{|c|}{ toluene + water } \\
\hline methyl methanesulfonate & 41 & 47 & 58 & 94 \\
\hline ethyl methanesulfonate & 19 & 21 & 27 & 68 \\
\hline n-Propyl methanesulfonate & 8 & 8 & 10 & 25 \\
\hline isopropyl methanesulfonate & 57 & 77 & 94 & 99 \\
\hline
\end{tabular}




\begin{tabular}{|l|r|r|r|r|}
\hline methyl benzenesulfonate & 5 & 5 & 6 & 14 \\
\hline n-Propyl benzenesulfonate & 1 & 2 & 2 & 8 \\
\hline isopropyl benzenesulfonate & 9 & 10 & 15 & 38 \\
\hline
\end{tabular}

\begin{tabular}{|l|r|r|r|r|}
\hline \multicolumn{5}{|c|}{ 2MeTHF alone } \\
\hline methyl methanesulfonate & 0 & 0 & 0 & 0 \\
\hline ethyl methanesulfonate & 0 & 0 & 0 & 0 \\
\hline n-Propyl methanesulfonate & 0 & 0 & 0 & 0 \\
\hline isopropyl methanesulfonate & 0 & 0 & 0 & 0 \\
\hline methyl benzenesulfonate & 0 & 0 & 0 & 0 \\
\hline n-Propyl benzenesulfonate & 0 & 0 & 0 & 0 \\
\hline isopropyl benzenesulfonate & 0 & 0 & 0 & 0
\end{tabular}

\begin{tabular}{|l|r|r|r|r|}
\hline \multicolumn{5}{|c|}{ THF alone } \\
\hline methyl methanesulfonate & 0 & 0 & 0 & 0 \\
\hline ethyl methanesulfonate & 0 & 0 & 0 & 0 \\
\hline n-Propyl methanesulfonate & 0 & 0 & 0 & 0 \\
\hline isopropyl methanesulfonate & 0 & 0 & 0 & 0 \\
\hline methyl benzenesulfonate & 0 & 0 & 0 & 0 \\
\hline n-Propyl benzenesulfonate & 0 & 0 & 0 & 0 \\
\hline isopropyl benzenesulfonate & 0 & 0 & 0 & 0
\end{tabular}

\begin{tabular}{|l|r|r|r|r|}
\hline \multicolumn{5}{|c|}{ NMP alone } \\
\hline methyl methanesulfonate & 0 & 0 & 0 & 11 \\
\hline ethyl methanesulfonate & 0 & 0 & 0 & 0 \\
\hline n-Propyl methanesulfonate & 0 & 0 & 0 & 0 \\
\hline isopropyl methanesulfonate & 0 & 0 & 0 & 0 \\
\hline methyl benzenesulfonate & 0 & 15 & 27 & 80 \\
\hline n-Propyl benzenesulfonate & 0 & 0 & 0 & 18 \\
\hline isopropyl benzenesulfonate & 0 & 0 & 0 & 27
\end{tabular}

\begin{tabular}{|l|r|r|r|r|}
\hline \multicolumn{5}{|c|}{ MeCN alone } \\
\hline methyl methanesulfonate & 2 & 1 & 4 & 6 \\
\hline ethyl methanesulfonate & 1 & 0 & 0 & 1 \\
\hline n-Propyl methanesulfonate & 0 & 0 & 0 & 0 \\
\hline isopropyl methanesulfonate & 0 & 0 & 0 & 0 \\
\hline
\end{tabular}




\begin{tabular}{|l|r|r|r|r|}
\hline methyl benzenesulfonate & 5 & 4 & 10 & 17 \\
\hline n-Propyl benzenesulfonate & 0 & 0 & 0 & 0 \\
\hline isopropyl benzenesulfonate & 0 & 0 & 0 & 0 \\
\hline
\end{tabular}

\begin{tabular}{|l|r|r|r|r|}
\hline \multicolumn{5}{|c|}{ EtOH alone } \\
\hline methyl methanesulfonate & 3 & 3 & 6 & 34 \\
\hline ethyl methanesulfonate & 2 & 1 & 1 & 14 \\
\hline n-Propyl methanesulfonate & 1 & 0 & 0 & 7 \\
\hline isopropyl methanesulfonate & 2 & 1 & 5 & 33 \\
\hline methyl benzenesulfonate & 6 & 5 & 13 & 57 \\
\hline n-Propyl benzenesulfonate & 0 & 0 & 0 & 13 \\
\hline isopropyl benzenesulfonate & 0 & 0 & 3 & 58
\end{tabular}

\begin{tabular}{|l|r|r|r|r|}
\hline \multicolumn{5}{|c|}{ EtOAc alone } \\
\hline methyl methanesulfonate & 2 & 1 & 4 & 9 \\
\hline ethyl methanesulfonate & 2 & 1 & 4 & 9 \\
\hline n-Propyl methanesulfonate & 1 & 0 & 2 & 7 \\
\hline isopropyl methanesulfonate & 1 & 0 & 3 & 8 \\
\hline methyl benzenesulfonate & 2 & 1 & 6 & 9 \\
\hline n-Propyl benzenesulfonate & 0 & 0 & 0 & 3 \\
\hline isopropyl benzenesulfonate & 0 & 0 & 0 & 1
\end{tabular}

\begin{tabular}{|l|r|r|r|r|}
\hline \multicolumn{5}{|c|}{ MTBE alone } \\
\hline methyl methanesulfonate & 4 & 6 & 8 & 28 \\
\hline ethyl methanesulfonate & 3 & 3 & 2 & 18 \\
\hline n-Propyl methanesulfonate & 3 & 2 & 2 & 17 \\
\hline isopropyl methanesulfonate & 2 & 2 & 2 & 17 \\
\hline methyl benzenesulfonate & 4 & 4 & 6 & 24 \\
\hline n-Propyl benzenesulfonate & 2 & 2 & 3 & 19 \\
\hline isopropyl benzenesulfonate & 2 & 3 & 5 & 20
\end{tabular}

\begin{tabular}{|l|r|r|r|r|}
\hline \multicolumn{5}{|c|}{ toluene alone } \\
\hline methyl methanesulfonate & 1 & 2 & 3 & 5 \\
\hline ethyl methanesulfonate & 0 & 0 & 1 & 1 \\
\hline n-Propyl methanesulfonate & 0 & 0 & 1 & 0 \\
\hline
\end{tabular}




\begin{tabular}{|l|r|r|r|r|}
\hline isopropyl methanesulfonate & 0 & 1 & 1 & 1 \\
\hline methyl benzenesulfonate & 1 & 1 & 2 & 4 \\
\hline n-Propyl benzenesulfonate & 0 & 0 & 2 & 3 \\
\hline isopropyl benzenesulfonate & 0 & 2 & 4 & 5 \\
\hline
\end{tabular}

\section{NMR Characterization of Alkylated DABCO Species}

All spectra were collected on an AV4400N Avance NEO 400z NMR Spectrometer NANO. NMR solvent peak residual internal DMSO-d ${ }_{6} \delta 2.51$.

1-methyl-1,4-diazabicyclo[2.2.2]octan-1-ium 


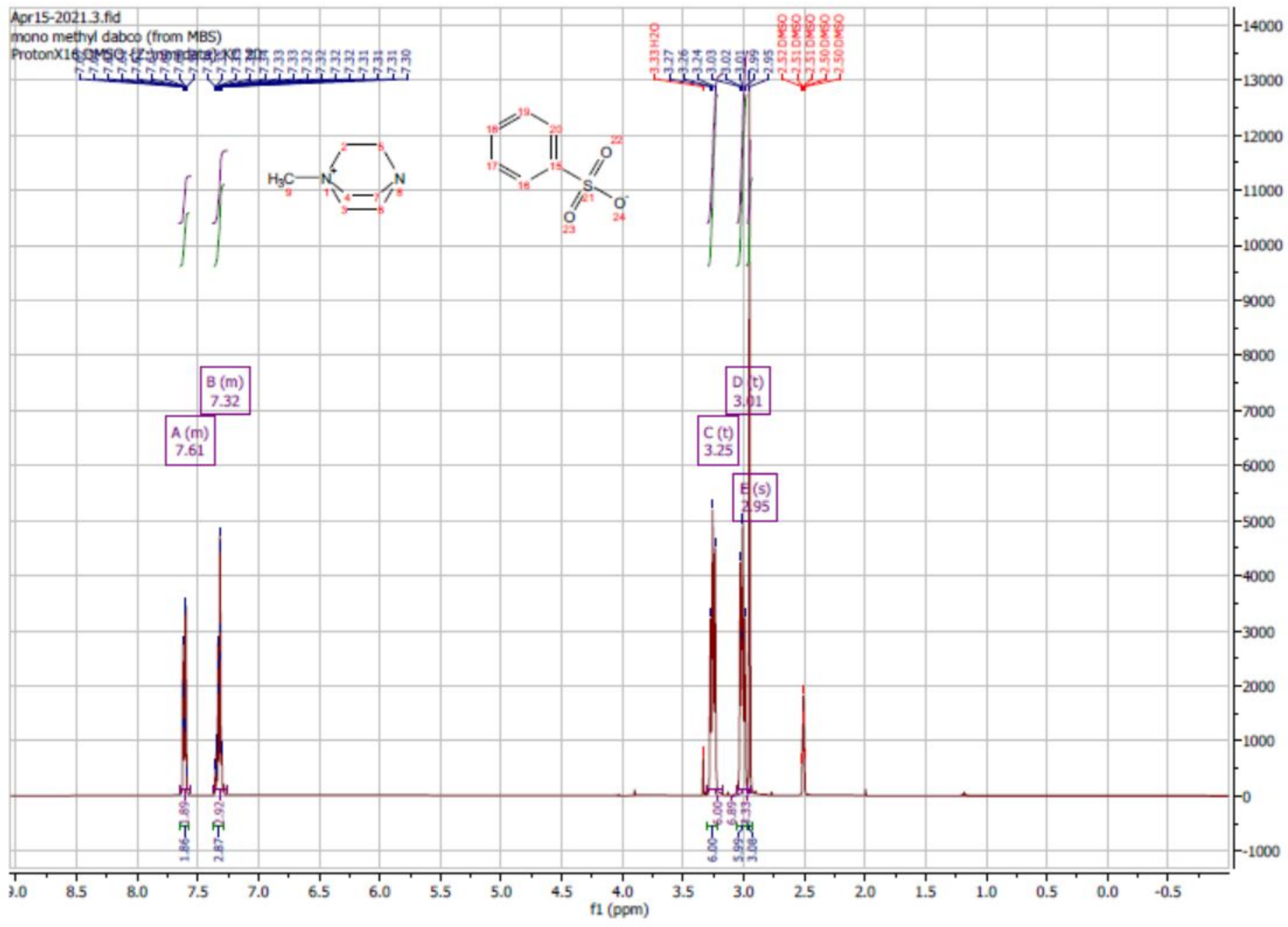

${ }^{1} \mathrm{H}$ NMR (400 MHz, DMSO- $\left.d_{6}\right) \delta 7.66-7.56(\mathrm{~m}, 2 \mathrm{H}), 7.38-7.26(\mathrm{~m}, 3 \mathrm{H}), 3.25(\mathrm{t}, 6 \mathrm{H}), 3.01(\mathrm{t}, J=9.1,6.1 \mathrm{~Hz}$, $7 \mathrm{H}), 2.95(\mathrm{~s}, 3 \mathrm{H})$.

1-ethyl-1,4-diazabicyclo[2.2.2]octan-1-ium 


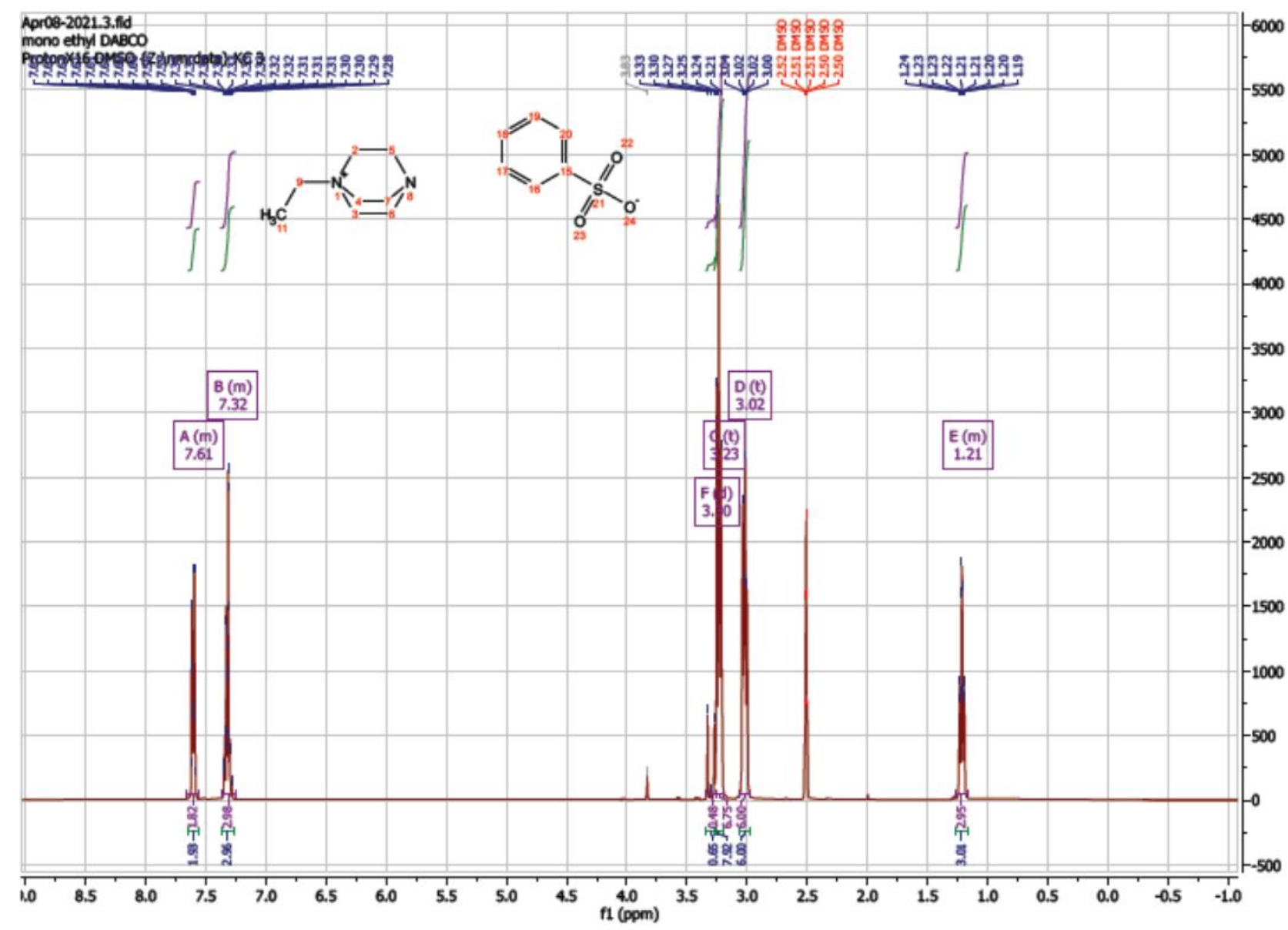

${ }^{1} \mathrm{H}$ NMR $\left(400 \mathrm{MHz}\right.$, DMSO- $\left.d_{6}\right) \delta 7.66-7.55(\mathrm{~m}, 2 \mathrm{H}), 7.38-7.26(\mathrm{~m}, 3 \mathrm{H}), 3.30(\mathrm{~d}, J=22.5 \mathrm{~Hz}, 0 \mathrm{H}), 3.23(\mathrm{t}, J=$ $7.6 \mathrm{~Hz}, 7 \mathrm{H}), 3.02(\mathrm{t}, J=8.9,6.0 \mathrm{~Hz}, 6 \mathrm{H}), 1.26-1.17(\mathrm{~m}, 3 \mathrm{H})$.

1-propyl-1,4-diazabicyclo[2.2.2]octan-1-ium 


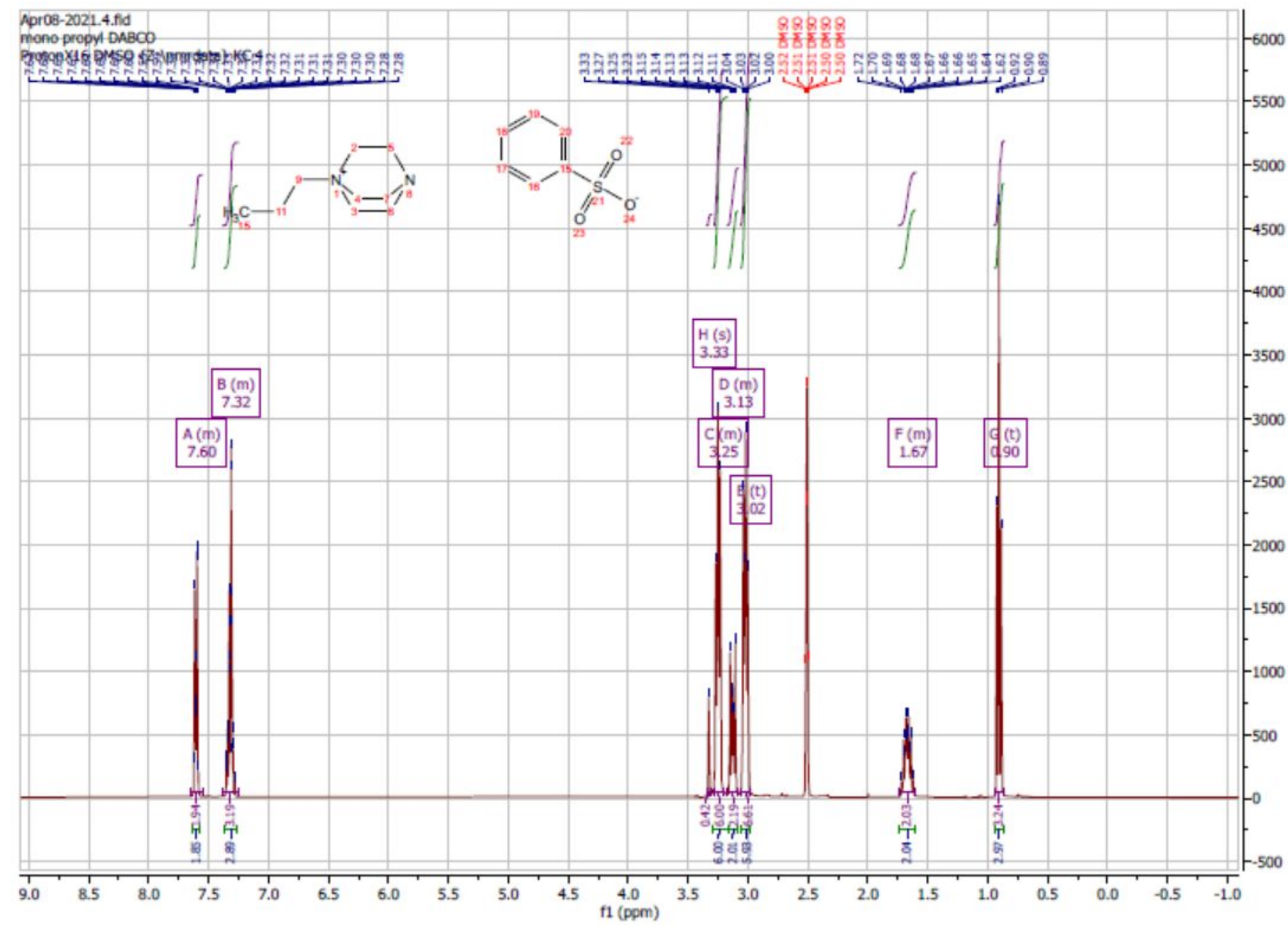

${ }^{1} \mathrm{H}$ NMR (400 MHz, DMSO- $\left.d_{6}\right) \delta 7.66-7.55(\mathrm{~m}, 2 \mathrm{H}), 7.38-7.25(\mathrm{~m}, 3 \mathrm{H}), 3.33(\mathrm{~s}, 0 \mathrm{H}), 3.28-3.21(\mathrm{~m}, 6 \mathrm{H}), 3.17$ $-3.08(\mathrm{~m}, 2 \mathrm{H}), 3.02(\mathrm{t}, J=8.9,6.1 \mathrm{~Hz}, 6 \mathrm{H}), 1.74-1.60(\mathrm{~m}, 2 \mathrm{H}), 0.90(\mathrm{t}, J=7.3 \mathrm{~Hz}, 3 \mathrm{H})$. 


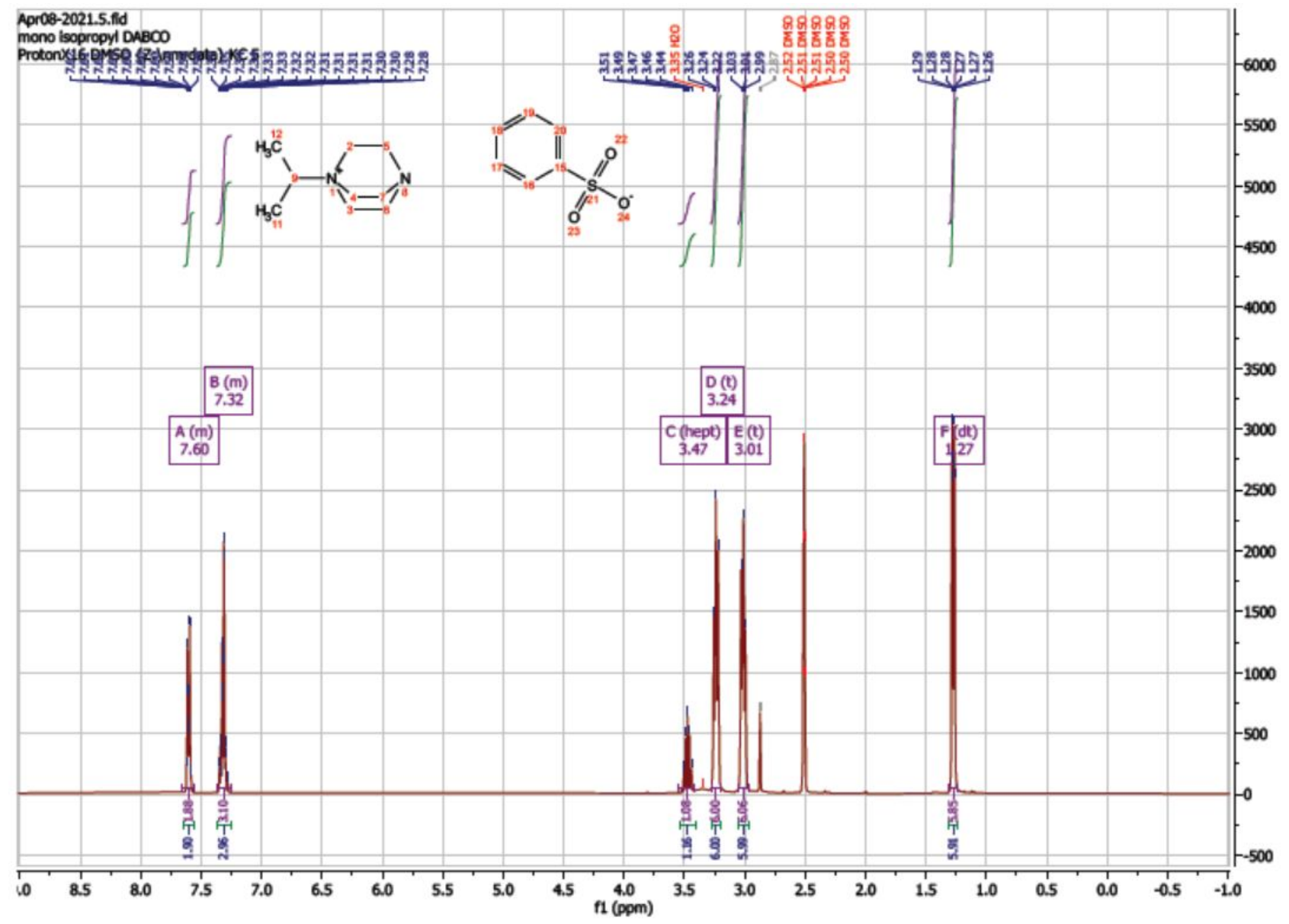

${ }^{1} \mathrm{H}$ NMR (400 MHz, DMSO- $d_{6}$ ) $\delta 7.65-7.55(\mathrm{~m}, 2 \mathrm{H}), 7.37-7.25(\mathrm{~m}, 3 \mathrm{H}), 3.47$ (hept, $\left.J=6.6 \mathrm{~Hz}, 1 \mathrm{H}\right), 3.24(\mathrm{t}$, $6 \mathrm{H}), 3.01(\mathrm{t}, J=7.3 \mathrm{~Hz}, 6 \mathrm{H}), 1.27(\mathrm{dt}, J=6.6,1.8 \mathrm{~Hz}, 6 \mathrm{H})$.

\section{LC/MS Data for Removal of Alkylated DABCO with Aqueous Washes}

The LC/MS method has a limit of detection for alkylated DABCO species of $\leq 10 \mathrm{ppb}$. Given the low solubility of these species in 2-MeTHF, preparation of a solution of known concentration was not practical and, therefore, a standard curve for solute quantitation was not prepared. However, when solutions were saturated with each alkyl DABCO species and analyzed, well-resolved peaks corresponding to each compound were observed. Following aqueous washes, peaks corresponding to each compound were either completely not detectable or extremely small, within the noise of the trace, accounting for a fraction of a percent of the initial (already low) concentration. It may therefore be concluded that the aqueous washes removed the alkylated DABCO species to the limit of detection. The below traces include the following data in the indicated order: 1) total ion trace for the given sample, 2) $\mathrm{M}+\mathrm{H}+$ for $\mathrm{DABCO}, 3$ ) expected $\mathrm{M}+\mathrm{H}+$ for mono methyl $\mathrm{DABCO}, 4$ ) expected $\mathrm{M}+\mathrm{H}+$ for mono ethyl $\mathrm{DABCO}, 5)$ expected $\mathrm{M}+\mathrm{H}+$ for $\mathrm{n}$-propyl and isopropyl $\mathrm{DABCO}$. 
Figure S9: Mono Methyl DABCO 2MeTHF Saturated Solution LC/MS Trace

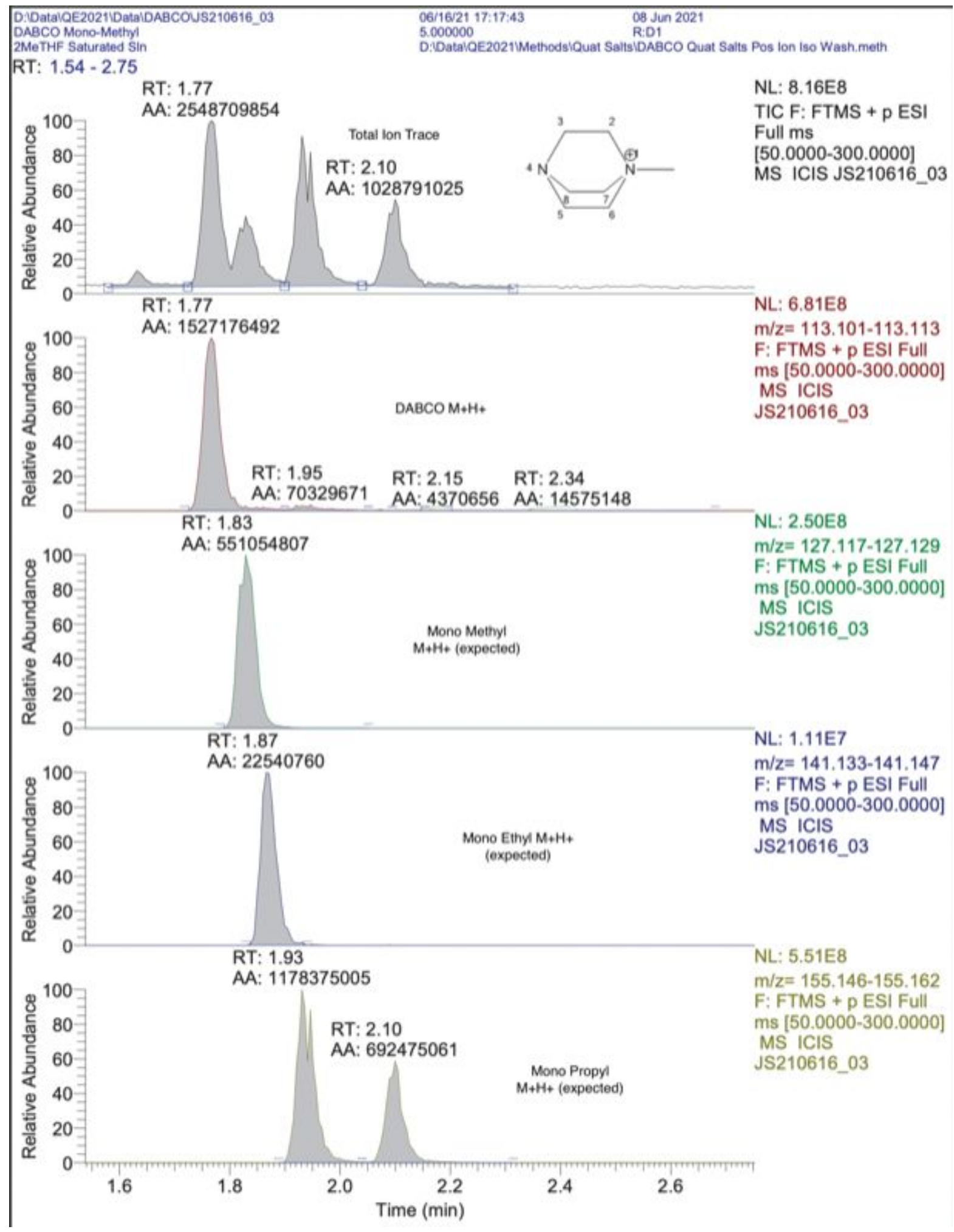


Figure S10: Mono Ethyl DABCO 2MeTHF Saturated Solution LC/MS Trace

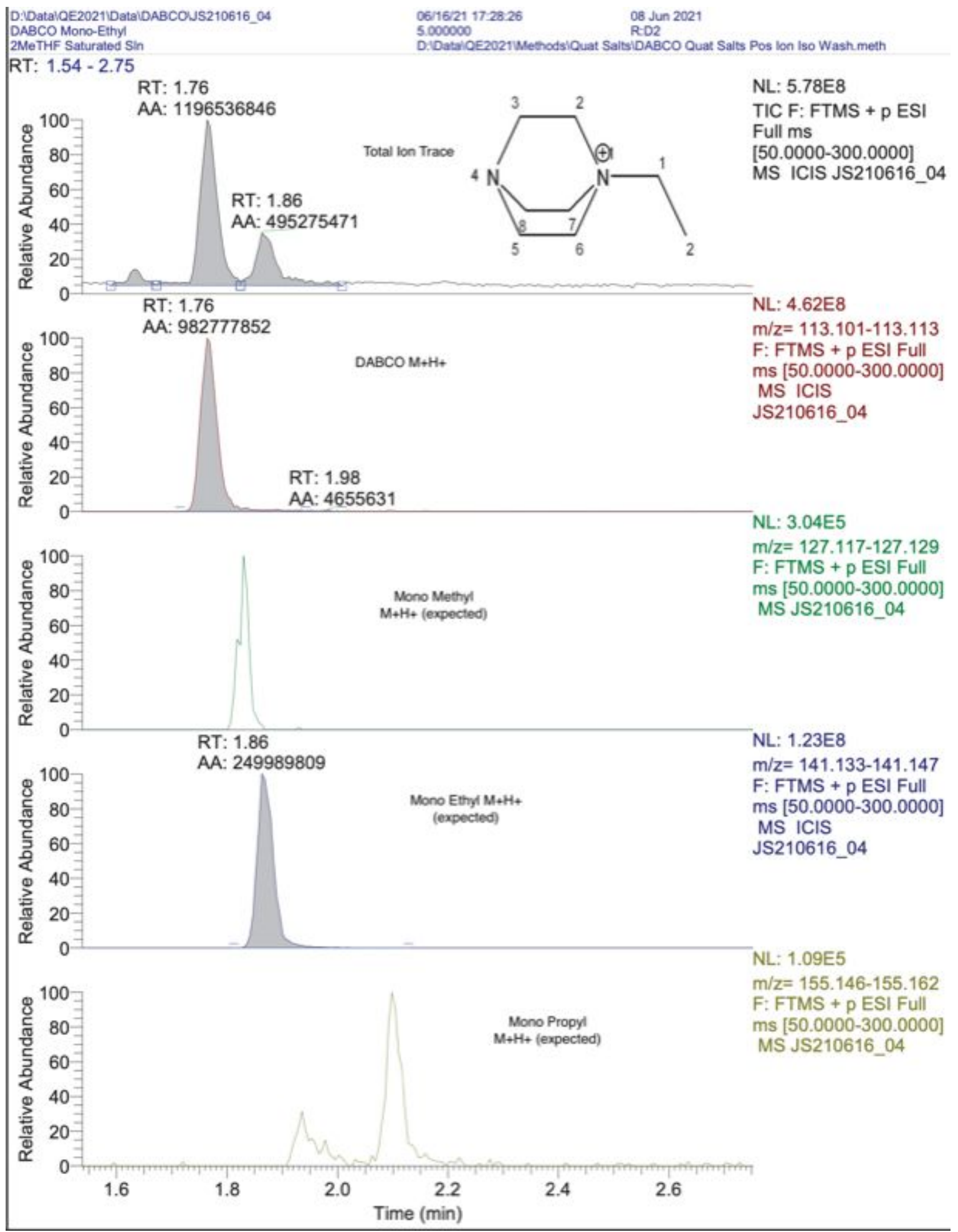


Figure S11: Mono $N$-Propyl DABCO 2MeTHF Saturated Solution LC/MS Trace

DiDatai OE2021DataiDABCOUS210616_05 DABCO Mono-Propy

RT: $1.54-2.75$

RT: 1.76

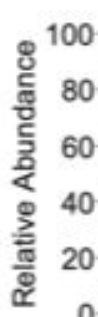

AA: 2348550636

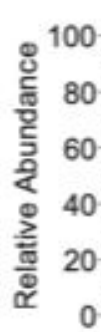

RT: 1.76

AA: 1850698493

100
80
60
40
20
0

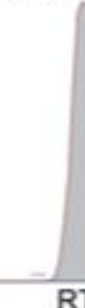

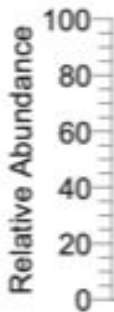

RT: 1.83

DABCO M+H*

RT: 2.09

AA: 1342210020

$06 / 16 / 2117: 39: 08$

5.000000

08 Jun 2021

RABCO Quat Salts Pos lon lso Wash meth

NL: $9.12 E 8$

TIC F: FTMS + p ESI

Full $\mathrm{ms}$

[50.0000-300.0000]

MS ICIS JS210616_05

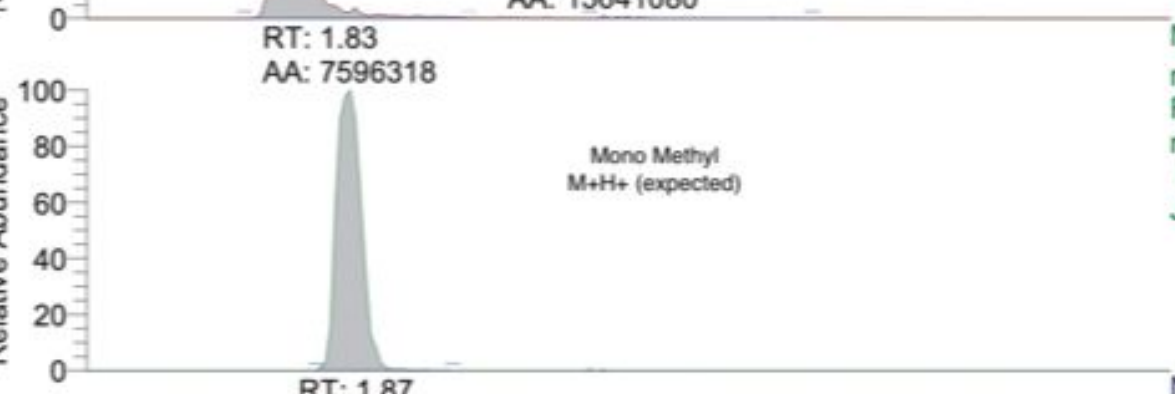

RT: 2.12

AA: 15641080
$\mathrm{NL}: 7.61 \mathrm{E} 8$

$\mathrm{m} / \mathrm{z}=113.101-113.113$

F: FTMS + p ESI Full ms [50.0000-300.0000] MS ICIS

JS210616_05
$\mathrm{NL}: 3.86 \mathrm{E} 6$

$\mathrm{m} / \mathrm{z}=127.117-127.129$

F: FTMS + p ESI Full ms [50.0000-300.0000] MS ICIS JS210616_05

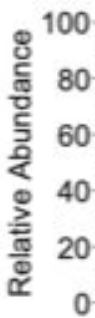

AA: 1051164

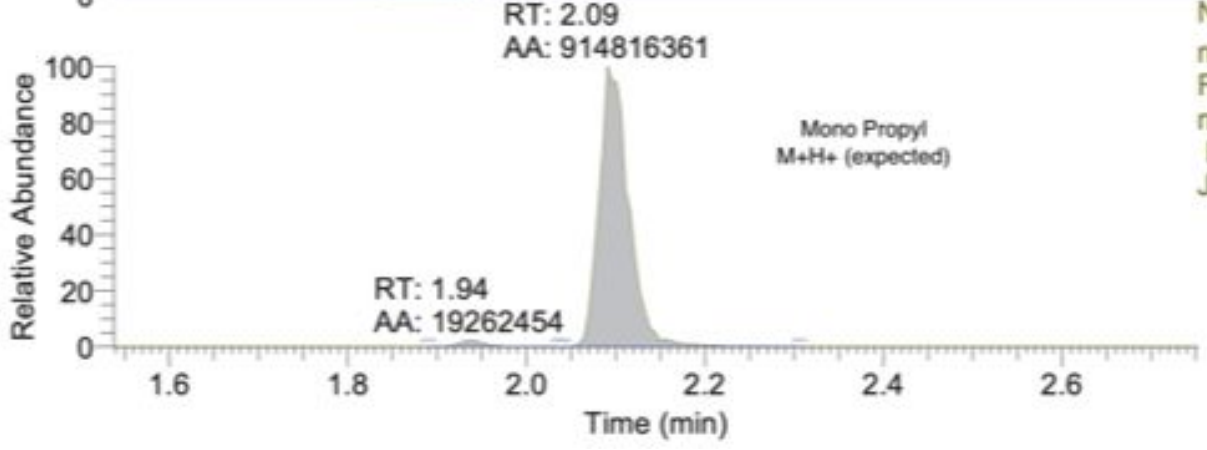

NL: $7.02 E 5$

$\mathrm{m} / \mathrm{z}=141.133-141.147$ F: FTMS + p ESI Full $\mathrm{ms}[50.0000-300.0000]$ MS ICIS JS210616_05

NL: 3.97E8

$\mathrm{m} / \mathrm{z}=155.146-155.162$ F: FTMS + p ESI Full ms [50.0000-300.0000] MS ICIS JS210616_05 
Figure S12: Mono isopropyl DABCO 2MeTHF Saturated Solution LC/MS Trace

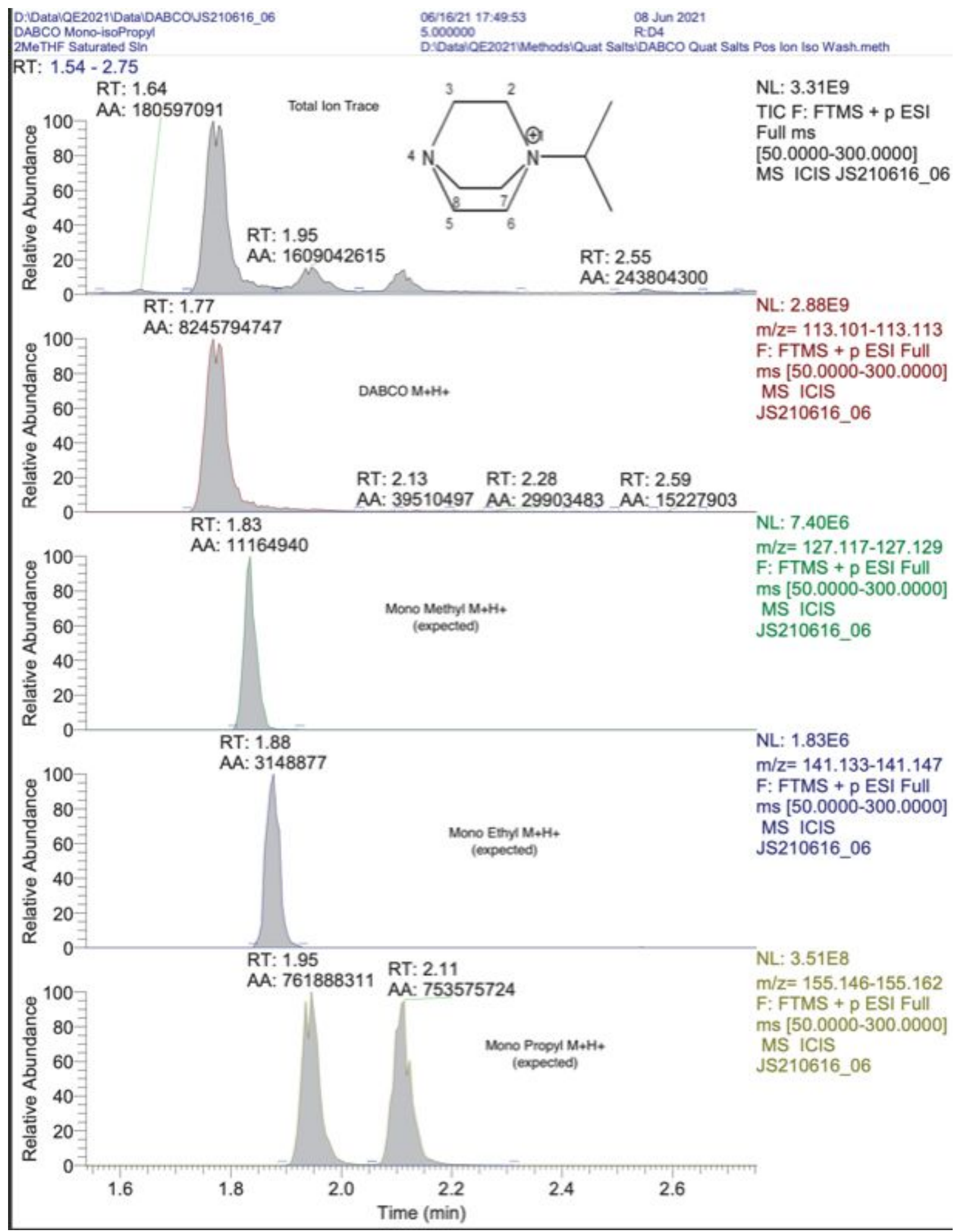


Figure S13: Mono Methyl DABCO Post-Water Wash 2MeTHF Solution LC/MS Trace

\begin{tabular}{|c|c|}
\hline $\begin{array}{l}\text { D:DatalOE20211DataiDABCOUS210616_13 } \\
\text { DABCO Mono-Methyl WW }\end{array}$ & $\begin{array}{ll}06 / 16 / 2119.05: 07 & \text { OS Jun } 2021 \\
5.000000 & \text { R:E1 }\end{array}$ \\
\hline Post Water Wash & D:DataVEe2021 MethodsiQuat SaltsiDABCO Quat Salts Pos Ion Iso Wa \\
\hline
\end{tabular}

Post Water Wash

DVatalOE2021WethodsiQuat Salts VDABCO Quat Salts Pos Ion iso Wash.meth

RT: $1.54-2.75$

RT: 1.64
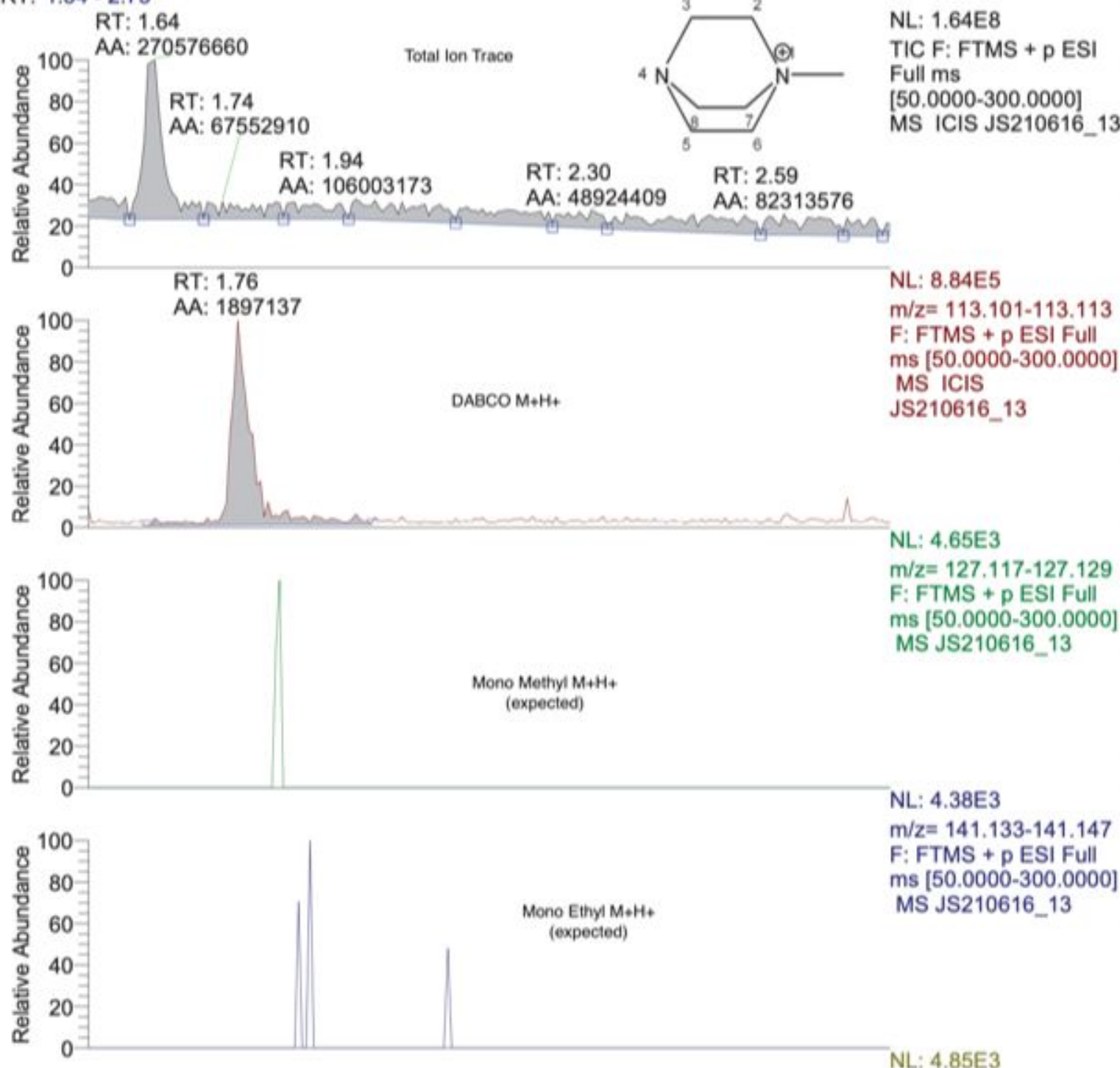

$\mathrm{m} / \mathrm{z}=141.133-141.147$

F: FTMS + p ESI Full ms [50.0000-300.0000] MS JS210616_13

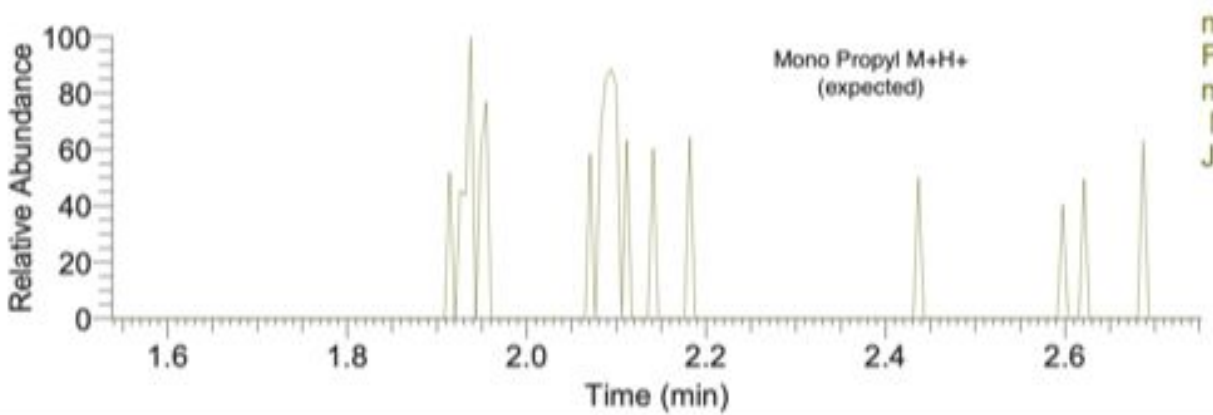

NL: $4.85 E 3$

$\mathrm{m} / \mathrm{z}=155,146-155.162$

F: FTMS + p ESI Full

$\mathrm{ms}[50.0000-300.0000]$

MS ICIS

JS210616_13 
Figure S14: Mono Ethyl DABCO Post-Water Wash 2MeTHF Solution LC/MS Trace

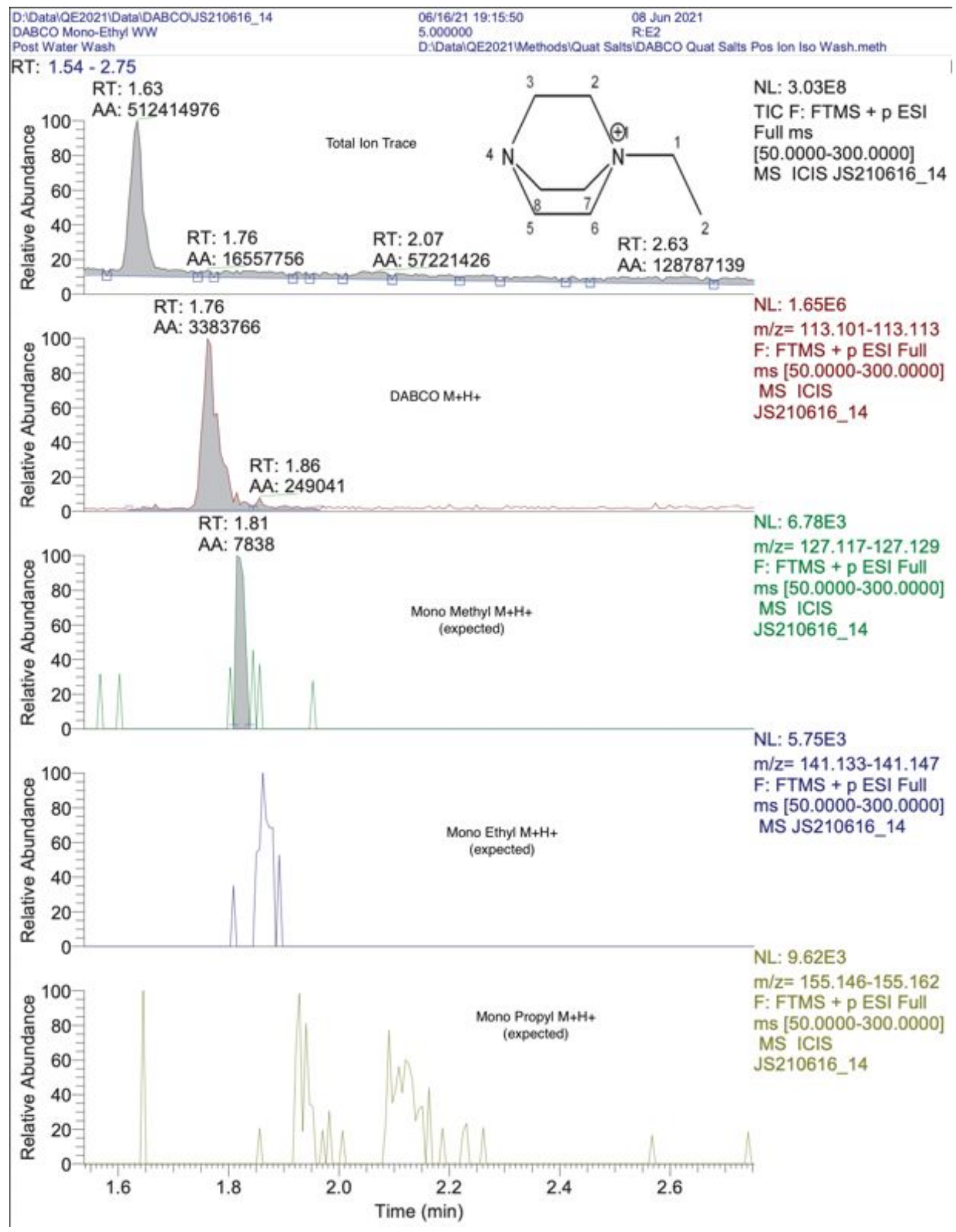


Figure S15: Mono N-Propyl DABCO Post-Water Wash 2MeTHF Solution LC/MS Trace

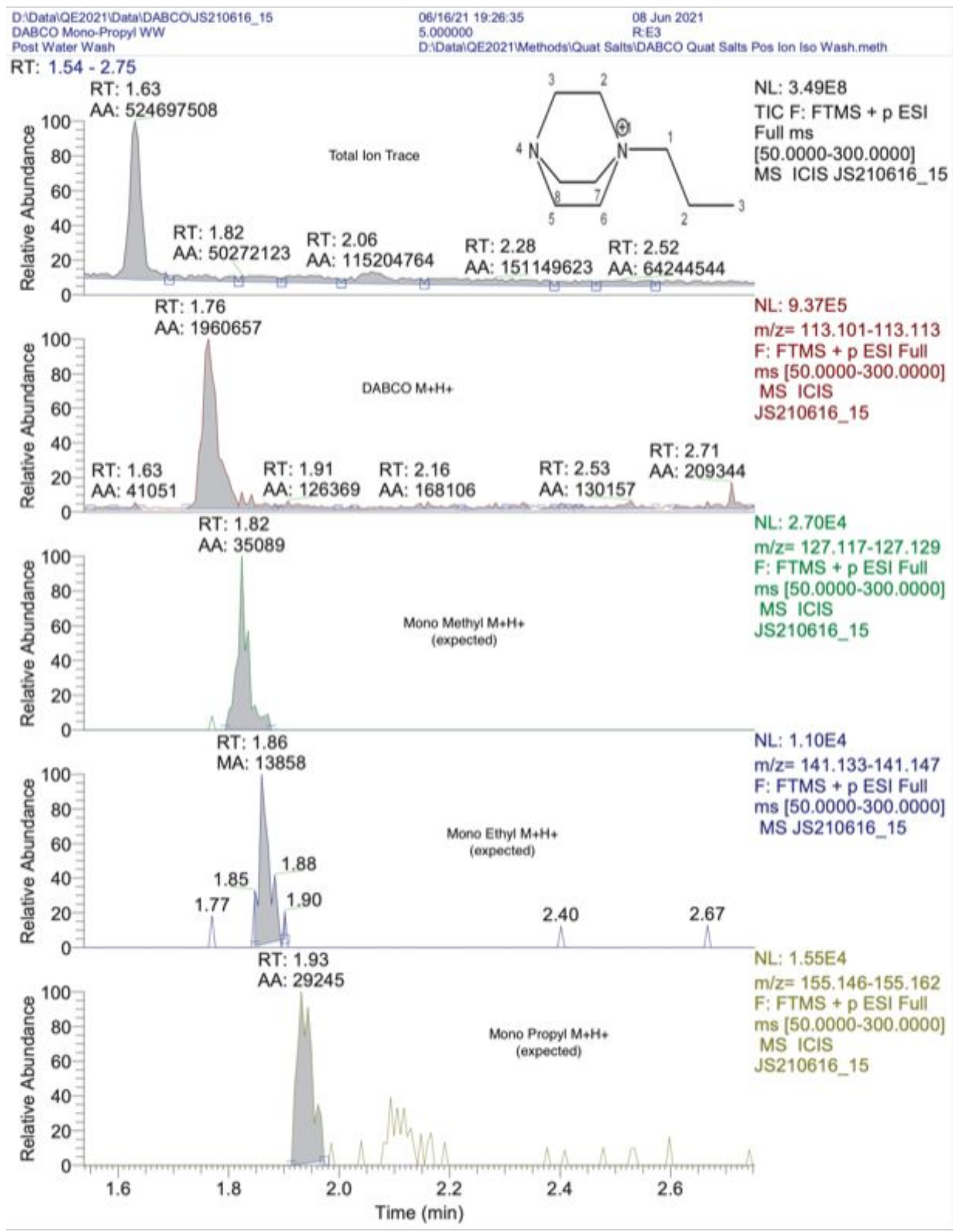


Figure S16: Mono Isopropyl DABCO Post-Water Wash 2MeTHF Solution LC/MS Trace

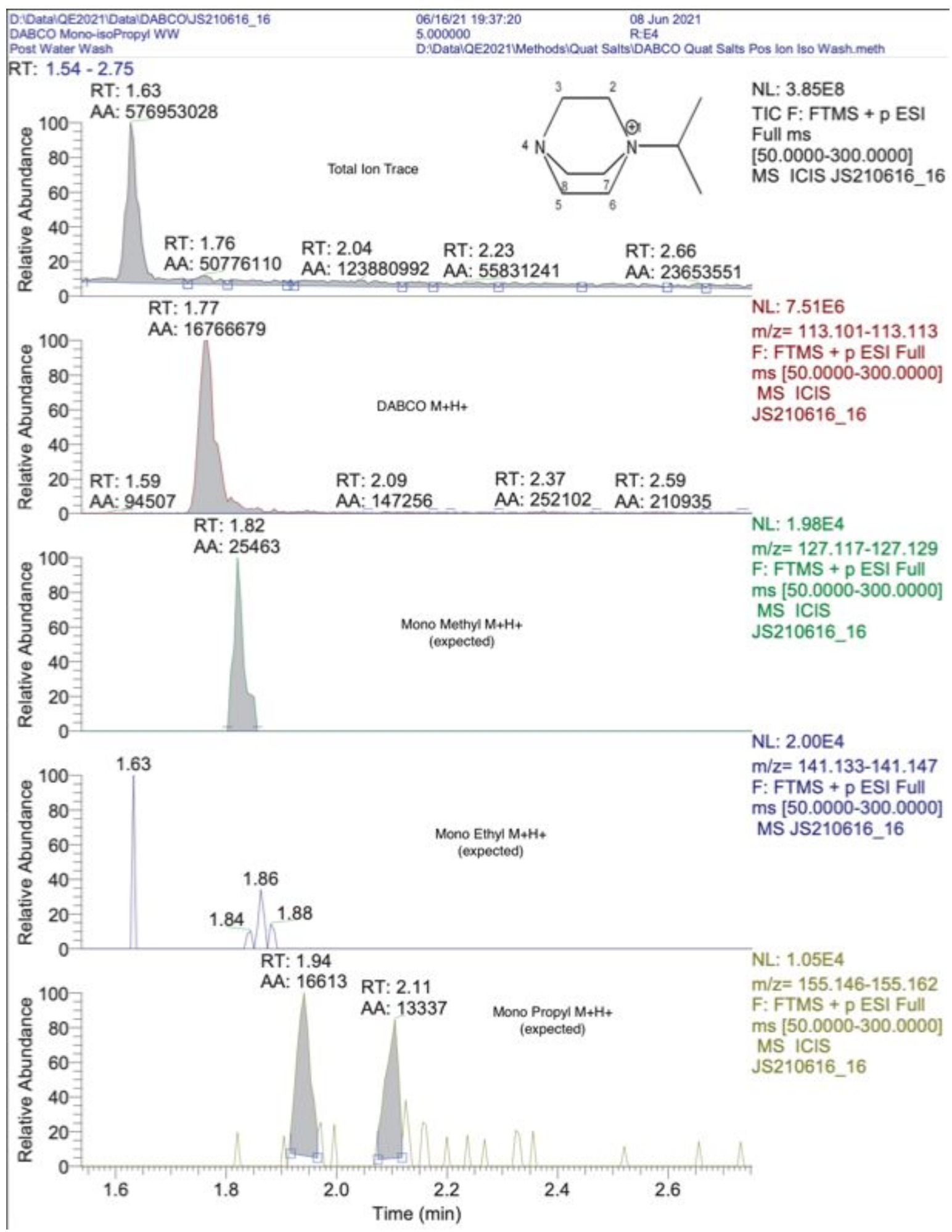


Figure S17: Mono Methyl DABCO Post-2N HCl Wash 2MeTHF Solution LC/MS Trace

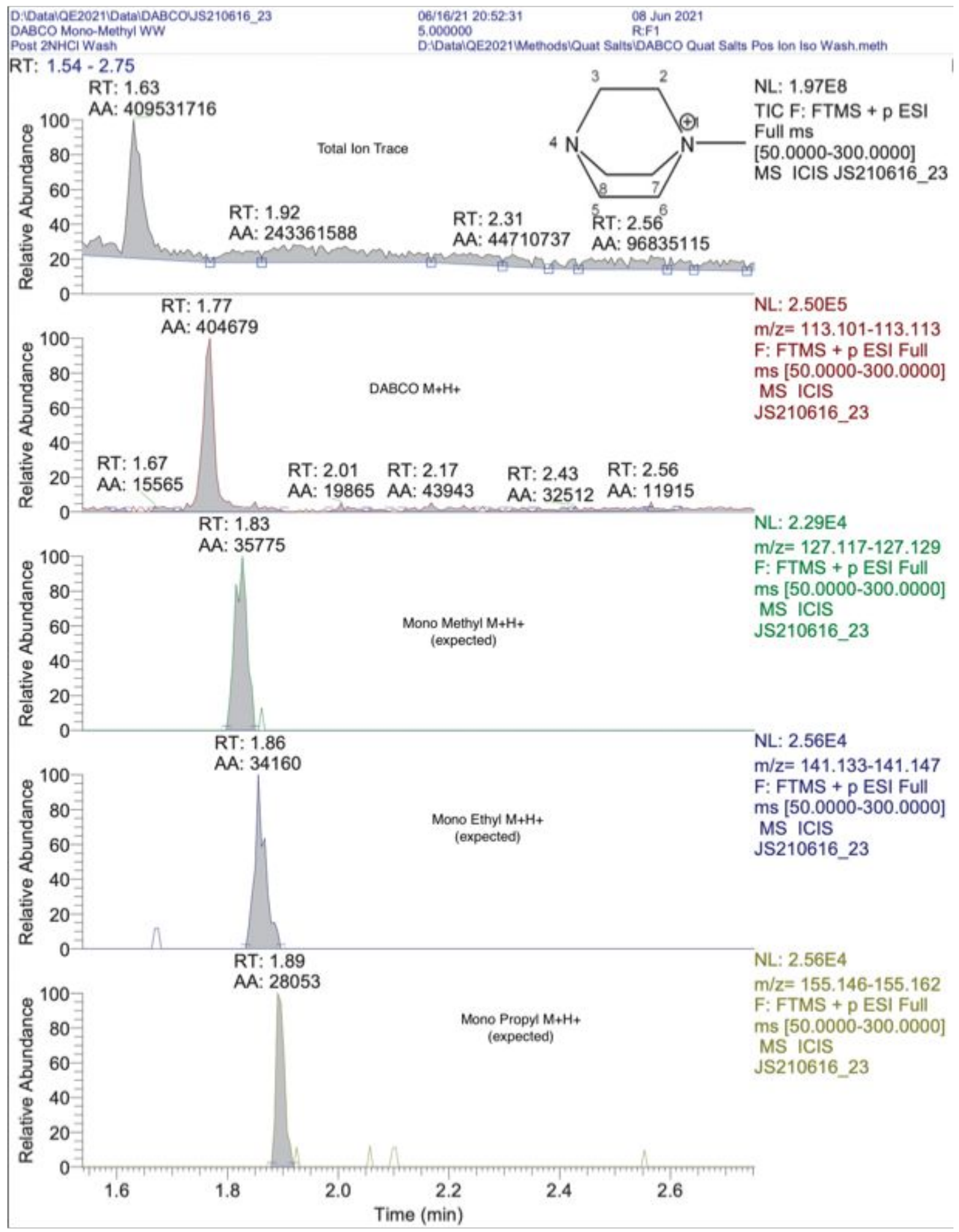


Figure S18: Mono Ethyl DABCO Post-2N HCl Wash 2MeTHF Solution LC/MS Trace

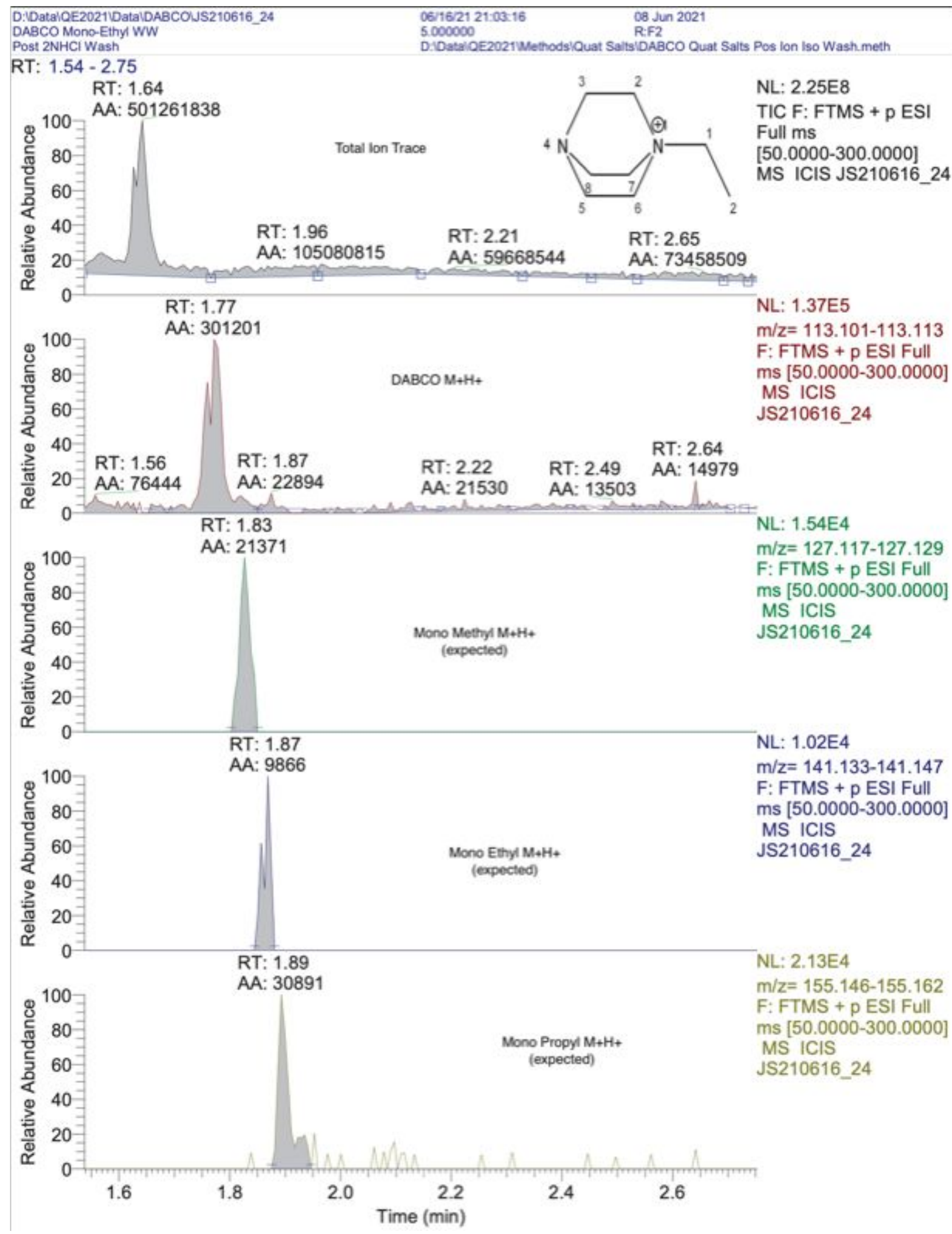


Figure S19: Mono $\mathrm{N}$-Propyl DABCO Post-2N HCl Wash 2MeTHF Solution LC/MS Trace

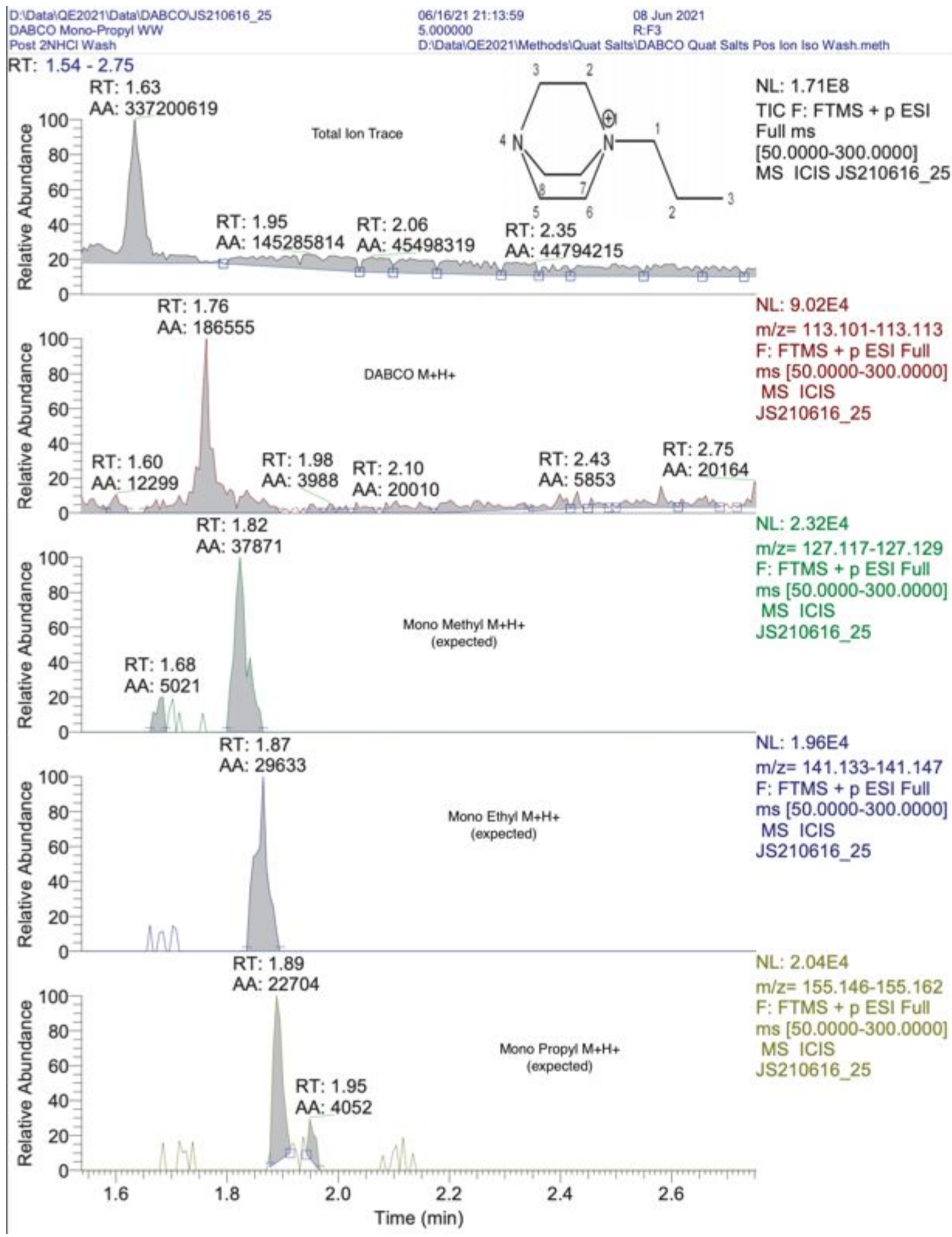


Figure S20: Mono Isopropyl DABCO Post-2N HCl Wash 2MeTHF Solution LC/MS Trace

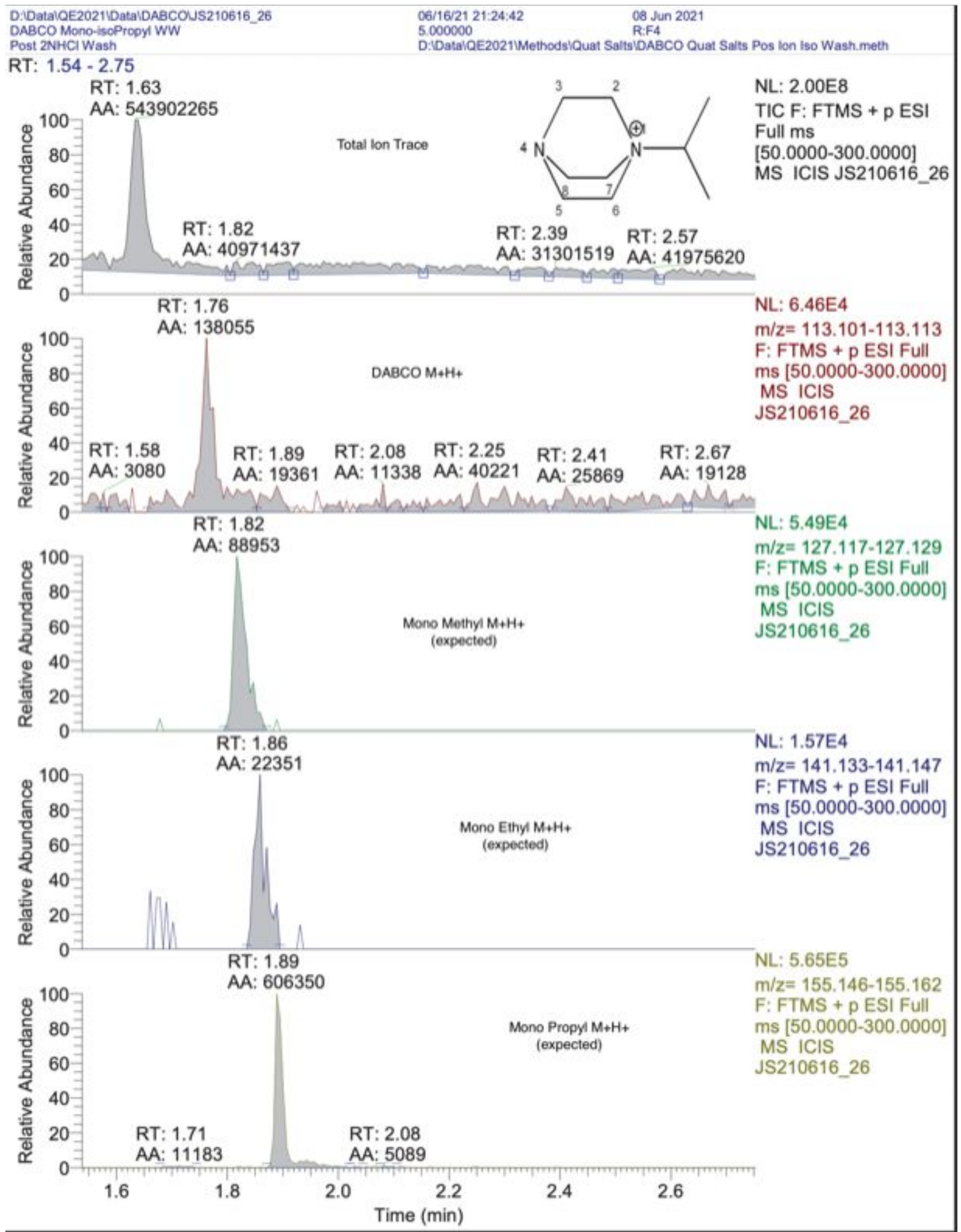

